\title{
Article
}

\section{A Particle Swarm Algorithm Based on a Multi-Stage Search Strategy}

\author{
Yong Shen, Wangzhen Cai, Hongwei Kang $* \mathbb{D}$, Xingping Sun, Qingyi Chen and Haigang Zhang \\ School of Software, Yunnan University, Kunming 650504, China; sheny@ynu.edu.cn (Y.S.); \\ caiwangzhen1@mail.ynu.edu.cn (W.C.); sunxp@ynu.edu.cn (X.S.); devas9@ynu.edu.cn (Q.C.); \\ portzhang@mail.ynu.edu.cn (H.Z.) \\ * Correspondence: hwkang@ynu.edu.cn
}

check for updates

Citation: Shen, Y.; Cai, W.; Kang, H.; Sun, X.; Chen, Q.; Zhang, H. A Particle Swarm Algorithm Based on a Multi-Stage Search Strategy. Entropy 2021, 23, 1200. https://doi.org/ $10.3390 /$ e23091200

Academic Editors: Giulia De Masi and Eliseo Ferrante

Received: 10 August 2021

Accepted: 9 September 2021

Published: 11 September 2021

Publisher's Note: MDPI stays neutral with regard to jurisdictional claims in published maps and institutional affiliations.

Copyright: (c) 2021 by the authors. Licensee MDPI, Basel, Switzerland. This article is an open access article distributed under the terms and conditions of the Creative Commons Attribution (CC BY) license (https:// creativecommons.org/licenses/by/ $4.0 /)$.

\begin{abstract}
Particle swarm optimization (PSO) has the disadvantages of easily getting trapped in local optima and a low search accuracy. Scores of approaches have been used to improve the diversity, search accuracy, and results of PSO, but the balance between exploration and exploitation remains sub-optimal. Many scholars have divided the population into multiple sub-populations with the aim of managing it in space. In this paper, a multi-stage search strategy that is dominated by mutual repulsion among particles and supplemented by attraction was proposed to control the traits of the population. From the angle of iteration time, the algorithm was able to adequately enhance the entropy of the population under the premise of satisfying the convergence, creating a more balanced search process. The study acquired satisfactory results from the CEC2017 test function by improving the standard PSO and improved PSO.
\end{abstract}

Keywords: particle swarm optimization; entropy; strategy; exploration and exploitation; repulsion and attraction

\section{Introduction}

Scholars have applied different approaches to the increasing number of structurally complex optimization problems, which are difficult to solve using traditional means, including evolutionary algorithms such as the genetic algorithm (GA) [1], bee colony (ABC) algorithm [2], difference (DE) algorithm [3], simulated annealing (SA) [4], ant colony (ACO) algorithm [5], and PSO [6].

PSO was proposed by Kennedy and Eberhart [7] in 1995 as a population-based heuristic optimization algorithm. With the advantages of a simple implementation, high efficiency, and few parameters, it is widely used in fields such as path planning [8], image segmentation [9], neural networks [10,11], data prediction [12], and noise control [13]. However, PSO is prone to getting trapped in local optima and lacks search accuracy in late iterations. To address these issues, PSO has been improved from four main points of view.

(1) Parameter tuning. Gunasundari et al. [14] proposed velocity-bounded Boolean PSO (VbBoPSO) based on binary PSO (BPSO), in which particles are initialized with random binary positions and velocities, with the velocity constrained to a specific range of values [15], to explore more regions and obtain better convergence. Sen et al. [16] proposed adaptive modified particle velocity PSO (MPV-PSO). A method to balance the search capability of PSO was proposed, using a strategy of linearly reducing the inertia weights [17]. Ratnaweera et al. [18] proposed a self-organizing hierarchical particle swarm optimizer with time-varying acceleration coefficients (HPSO-TVAC), which varies the acceleration coefficients to improve the search capabilities. Zhan et al. [19] proposed adaptive particle swarm optimization (APSO) with adaptive control parameters. Chen et al. [20] proposed chaotic dynamic weight particle swarm optimization (CDW-PSO), using inertia quantities with chaotic mapping to modify the search direction.

(2) Learning strategy. PSO is prone to getting trapped in local optima, and the search accuracy is not sufficient in late iterations because it uses only two experiences to 
guide particle learning. Improving this aspect of the learning strategy has attracted much attention. Liang et al. [21] proposed a dynamic multi-swarm particle swarm optimizer (DMS-PSO) with a dynamic neighborhood structure, in which the learning of each particle is no longer restricted to one population. Liu et al. [22] proposed a hierarchical simple time hierarchy strategy (THSPSO) algorithm using different learning strategies in different search phases. Zhan et al. [23] proposed orthogonal learning particle swarm optimization (OLPSO) with orthogonal learning, in which each particle obtains useful information from its own historical best experience and that of its neighbors. Xu et al. [24] proposed twoswarm learning particle swarm optimization (TSLPSO) based on dimensional learning, which constructs a learning paradigm for each particle by learning each dimension of its individual optimal position from the corresponding dimension of the population optimal position. Finally, Li et al. [25] proposed a learning strategy based on the collaboration of multiple populations to achieve information sharing and co-evolution among populations.

(3) Topology. Kennedy [26] pointed out that the use of topology is effective for population-based algorithms. In structured populations, information is often exchanged between closely linked individuals based on fitness and topological relationships as a way to slow down convergence. Mendes et al. [27] proposed fully informed particle swarm optimization (FIPSO), which uses a fully informed strategy in which each particle is updated based on the historical best experience of its neighbors. Janson et al. [28] constructed a dynamically changing tree topology in which each particle learns from its parent, effectively using the information of each particle.

(4) Algorithm crossover. Hybrid algorithms are a key research area to improve the performance of PSO algorithms. They incorporate operators such as crossover, selection, mutation, and choice to improve the search quality of the population individuals and the general efficiency of the algorithm. For example, the use of genetic operators can improve population diversity and convergence to the global optimum. Hybrid algorithms can better escape local optima and overcome certain inherent drawbacks associated with single algorithms. Zhang et al. [29] proposed differential mutation and novel social learning PSO (DSPSO), which combined four differential variation operations with social learning particle swarm optimization (SLPSO). Nasiraghdam et al. [30] proposed a new approach based on a hybrid genetic algorithm and PSO. Related studies [31-34] showed that a hybrid PSO algorithm incorporating other evolutionary algorithms not only improves population diversity but prevents premature convergence and increases the probability of finding a global optimal solution.

In summary, the key to PSO improvement lies in balancing diversity and convergence, preventing premature convergence to a local optimum, and improving local exploitation. Previous work used entropy as an evaluation index to improve the algorithm but stopped at using entropy to constrain the population traits and did not explore a more optimal search process. Others divided up the search strategy using both new strategies or vastly different ones. This paper proposes a multi-stage search strategy to fully maintain the population diversity for the global search in the early stage and modifies the update formula in the late stage to help jump out of the local optima, improving the local search ability. While improving the algorithm exploitation ability and search accuracy, the original algorithm converges after adding the strategy. This strategy can be used as an improved operator and implemented in heterogeneous comprehensive learning particle swarm optimization (HCLPSO) and TSLPSO. Both of these algorithms divide sub-populations to balance exploration and exploitation; however, adding a new balancing strategy can have adverse effects. This paper explores these two representative algorithms, which are compatible with the new strategy, to illustrate the effectiveness of the strategy. The experimental results show that this strategy can effectively improve the probability of the algorithm converging to the global optimal solution. 


\section{Related Knowledge}

\subsection{Particle Swarm Optimization}

PSO tends to a global convergence through the cooperation and competition of particles in the search space. The particle velocity and position updates are determined by the best position found by the corresponding particle (pbest) and the best position found by the whole population up to the current iteration of the algorithm (gbest). Let the total number of particles be $\mathrm{N}$, the dimension $\mathrm{D}$, and the maximum number of iterations maxiter. Then, the velocity of the $i$ th particle at moment $t$ is $v_{i}(t)=\left[v_{i 1}(t), v_{i 2}(t), \ldots, v_{i m}(t)\right]^{T}$, the position is $x_{i}(t)=\left[x_{i 1}(t), x_{i 2}(t), \ldots, x_{i m}(t)\right]^{T}$, the best position found by the corresponding particle at moment $t$ is pbest $t_{i}(t)=\left[p_{i 1}(t), p_{i 2}(t), \ldots, p_{i m}(t)\right]^{T}$, and the best position found by the whole population is gbest $=\left[g_{1}, g_{2}, \ldots, g_{m}\right]^{T}$. The particles are iteratively searched according to the following formula:

$$
\begin{gathered}
v_{i+1}(t+1)=\omega v_{i}(t)+c_{1} r_{1}\left(\text { pbest }_{i}(t)-x_{i}(t)\right)+c_{2} r_{2}\left(g b e s t-x_{i}(t)\right), \\
x_{i+1}(t+1)=x_{i}(t)+v_{i+1}(t+1),
\end{gathered}
$$

where the inertia weight coefficients are the learning factors. $r_{1}$ and $r_{2}$ are random numbers between [0,1]. The two factors $c_{1}$ and $c_{2}$, known as "acceleration coefficients", are positive constants commonly used to determine when the cognition speed of the $i$ th particle is accelerated towards pbest and gbest, respectively. The symbol $\omega$ denotes the inertia weight parameter which was originally developed to address the velocity explosion problem.

\subsection{TSLPSO Algorithm}

Currently, TSLPSO is one of the algorithms that achieves superior results on the congress on evolutionary computation (CEC) test function [24], in which one sub-population uses the learning paradigm constructed by the dimensional learning strategy to guide the local search of particles, and the other uses the learning paradigm constructed by the integrated learning strategy to guide the global search. The learning paradigm constructed by dimensional learning can guide the particles to search in better regions; however, if the majority of particles are near a local optimum and are trapped there, then premature convergence occurs. To solve this problem, the integrated learning strategy is introduced to enhance population diversity and help particles escape local optima.

\subsection{HCLPSO Algorithm}

In HCLPSO, the population is divided into two sub-populations. The first aims to enhance exploration and the second to enhance exploitation. In both sub-populations, samples are generated using an integrated learning (CL) strategy with learning probability Pc to generate random numbers for each dimension of the particles and to compare them with their respective learning probability $\mathrm{P}_{\mathrm{i}}$ values. The velocity of the exploration subpopulation is updated as

$$
V_{i}^{d}=\omega V_{i}^{d}+c * \operatorname{rand}_{i}^{d} *\left(\text { pbest }_{f_{i}(d)}^{d}-X_{i}^{d}\right),
$$

and the velocity of the exploitation sub-population as

$$
V_{i}^{d}=\omega V_{i}^{d}+c_{1} * \operatorname{rand}_{i}^{d} *\left(\operatorname{pbest}_{f_{i}(d)}^{d}-X_{i}^{d}\right)+c_{2} * \operatorname{rand} 2_{i}^{d} *\left(\text { gbest }^{d}-X_{i}^{d}\right) .
$$

where $f_{i}(d)=\left[f_{i}(1), f_{i}(2), \ldots, f_{i}(D)\right]$ indicates whether the i-th particle of each dimension $\mathrm{d}$ follows its own or another's pbest. When rand $d_{i}^{d}>\mathrm{Pc}_{\mathrm{i}}, f_{i}(d)=1$; when rand ${ }_{i}^{d}<\mathrm{Pc}_{\mathrm{i}}$, then two particles, namely $X_{j}$ and $X_{k}$, are randomly selected from the sub-population of the $i$ th particle. If fitness $\left(X_{j}\right) \leq$ fitness $\left(X_{k}\right)$, then $f_{i}(d)=j$; otherwise, $f_{i}(d)=k$.

Since the exploring particles are not allowed to access the information of the exploiting particles, there is no information flow from the exploiting sub-population to the exploring sub-population. To prevent loss of diversity, exploring and exploiting individ- 
uals do not interact. Inertia weights and dynamic acceleration coefficients are used for both sub-populations.

\section{Particle Swarm Optimization with a Multi-Stage Search Strategy}

\subsection{Multi-Stage Search Strategy}

The paper uses the multi-stage search strategy to improve the search results based on the original mechanisms of PSO, TSLPSO, and HCLPSO. The multi-stage search strategy is described below.

Before population iteration, each dimension of each particle is given an additional attribute Ra, the value of which is 1 or -1 . As shown in Figure 1, blue signifies that the Ra attribute value of the particle is 1 , and red signifies that the value of the particle is -1 . In subsequent action behavior judgments, this value determines whether two particles are mutually attracted or repelled, i.e., particles attract each other with the opposite Ra value, but repel each other if they are the same. In the first stage, a certain proportion of better adapted particles are selected, and these will not be attracted or repelled, as indicated by the black box at the edge of the particles in Figure 1. The remaining particles will be affected by the better adapted particle swarm, except for those that change their position according to the original algorithm, i.e., those without the black border in the figure, and the strength of the influence is determined by the parameter pow. To prevent the phenomenon of premature clustering, the Euclidean distance between particles is calculated as

$$
d\left(X_{i}, X_{j}\right)=\sqrt{\sum_{D=1}^{n}\left(X_{i}^{d}-X_{j}^{d}\right)^{2}} .
$$

when the distance is less than the threshold, the particles with poor fitness will be bounced away.

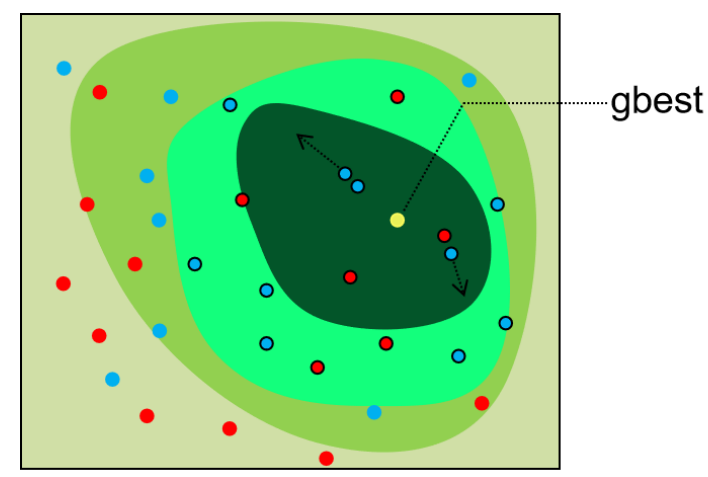

Figure 1. Particle swarm optimization in search strategy 1.

In the second stage, no operation is applied to the particle population. In the third stage, only the Ra of the optimal particle is kept unchanged, and all other particles have the opposite property value of the optimal particle in each dimension, i.e., they will be attracted by it.

In the velocity update Formula (1), particles are pulled by the gbest and pbest points. This seems similar to the attraction and repulsion of this strategy, but in fact, the PSO easily traps in local optima because particles are influenced by the current gbest point in the early stage, and the poor search accuracy in the later stage is caused by the attraction of the pbest point. Existing improvement algorithms make dynamic linear changes to learning factors $c_{1}$ and $c_{2}$ in the velocity update Formula (1) to achieve the expected improvement. However, from another perspective, the improvement strategy is independent of the velocity and position update Formulae (1) and (2), and this study sought to improve the algorithm in terms of exploration and exploitation and to control the shape of the population by relatively direct and simple means. From the analysis of the experimental results in Section 6, it can be seen that the algorithm's performance has a potential point of 
balance between exploration and exploitation, and thus a higher probability of obtaining the optimal solution.

\subsection{Definition of Particle Actions}

\subsubsection{Particle Action Definition for Stage 1}

The position and velocity of all particles are updated according to velocity and position update Formulate (1) and (2), the position of the lagging particles will change according to the fitness value of the particles, and the direction of movement is determined by the Ra value. The Ra value is an inherent property of each particle: its positive or negative value determines repulsion or attraction between particles, i.e., particles attract those with the opposite Ra value, but repel those with the same. Each particle has a Ra value for each dimension.

$$
R a_{i}^{d}=\left\{\begin{array}{lr}
-1 & \operatorname{rand}(0,1)>0.5 \\
1 & \text { else }
\end{array}\right.
$$

As a result of the randomness of $\mathrm{Ra}$, a lag particle action also has randomness. When the force value pow is large enough, the new interaction force between particles can dominate the movement of the lag particle swarm. The force value is calculated as

$$
\text { pow }=-\sum_{j=1}^{M} \sum_{d=1}^{D}\left(1-\frac{\left|X_{j}^{d}-X_{i}^{d}\right|}{\text { searchrange }}\right) * \frac{R a_{j}^{d}}{R a_{i}^{d}} * \text { str },
$$

where str is a constant parameter that determines pow, which changes the speed of the particle; the "searchrange" is limited by $X_{\min }$ and $X_{\max }$. The particle itself has a speed, which means that these particles may be pushed away from the gbest point, may be close to the gbest point, or may oscillate in its vicinity. However, since the size of the lag particle population is fixed, only a certain number of particles will act to make up for the difference. If a lag particle improves its fitness beyond that of other particles after iteration, then other particles will replace it as the lag particle population. Put differently, this mechanism ensures that part of the particle swarm is exploited locally, and the rest is explored globally; this study sought a balance between them.

To limit premature convergence, which is the tendency to trap in local optima, the Euclidean distance between particles is incorporated in stage 1. Once the distance between any two particles reaches a threshold, the particle with the lag fitness value will be selected and a force applied to it in the opposite direction with respect to the gbest point, pushing it away from the region in which it was originally located. This strategy coexists with the strategy repulsion and attraction mentioned above, and they affect each other.

\subsubsection{Particle Action Definition for Stage 2}

As shown in Figure 2, stage 2 is mainly used to judge the population traits and control the transition of the stage; in this study, no additional actions were performed on the particles. By mapping the diversity and convergence of the improvement algorithm, in this study, the parameters were set to control the end of stage 1 and the beginning of stage 3 . Stage 2 did not originally exist in our design, but it was found that the experimental results could be improved by incorporating stage 2 . Therefore, stage 2 could not be abandoned.

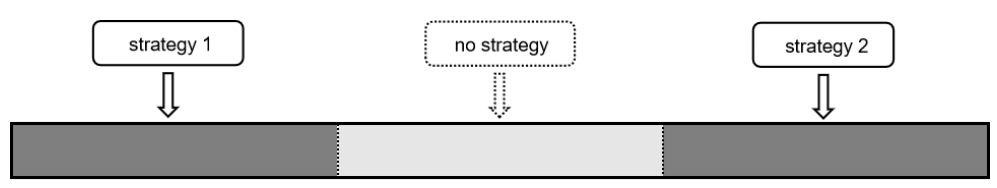

Figure 2. Axis diagram of population iteration number.

\subsubsection{Particle Action Definition for Stage 3}

In stage 3, we redefined the additional behavior of the particles, maintaining the Ra value of the current particle closest to the gbest point, and making the Ra values of all other 
particles the opposite, i.e., all other particles will be attracted by the particle near the gbest point, the power force decreases with the number of iterations, and it will decay to 0 at the end of the iteration.

$$
\text { pow }=\text { pow }-\frac{s t r * N}{E F S *(0.5-\text { stage })},
$$

where "stage" is the value of the parameter that divides the stage and EFS represents fitness evaluations.

It should be noted that the optimal particle may change, so the pow of each particle will be recalculated after each iteration, and there is no Ra influence between other particles except the optimal particle.

Unlike the standard PSO, in which particles are influenced by the information of the pbest and gbest points, in stage 3, particles are influenced by the information of the current optimal particle position, which does not overlap with the gbest point position. The subtle differences between the two can be seen in Figure 3. The addition of a new guidance factor can help improve the convergence performance of PSO, and such a definition reduces the problem of poor search accuracy caused by the traction of the pbest point in the late stage of the search. Moreover, the current optimal particle position and the gbest point jointly determine the search direction to improve convergence performance.

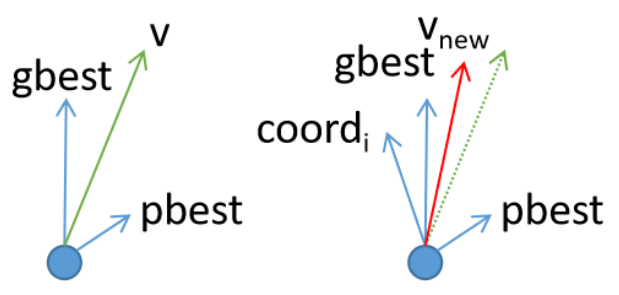

Figure 3. Particle velocity diagram in stage 3.

\subsection{Particle Property Change History}

Ra is randomly attached to each particle at the beginning of phase 1 and will change in the following situations:

(1) A particle distance determination is triggered in stage 1. The Ra of the less adapted of two particles will be reassigned with the same value as the other particle;

(2) At the beginning of stage 3, a particle that is not the best adapted will have an Ra value opposite to that of the best particle;

(3) When the optimal particle changes in stage 3, repeat 2.

\subsection{Inter-Particle Action}

The interactions between particles are summarized as follows, where repulsion and attraction are reflected by the change in the direction of velocity and magnitude, which directly acts on velocity change:

(1) In all phases, on the basis of the original algorithm velocity and position update formula, particles that are equivalent to each other will be subject to attraction from the gbest point;

(2) In stage 1, the lag particle will be attracted by the pow in Formula (7) from the better particle population;

(3) In stage 1, the particle with the most lag of two close particles will be subject to repulsive force;

(4) In stage 3, the non-optimal particle will be attracted by the optimal particle.

\subsection{Framework of RaPSO Algorithm}

Input: Tmax is the maximum number of iterations, and Dim is the number of dimensions, Ra: denotes additional properties, and pow denotes force parameters. 
Output: GbestValue is the global optimal solution, and a and b are constants for control phase division.

Figure 4 is the framework of the RaPSO algorithm (Algorithm 1).

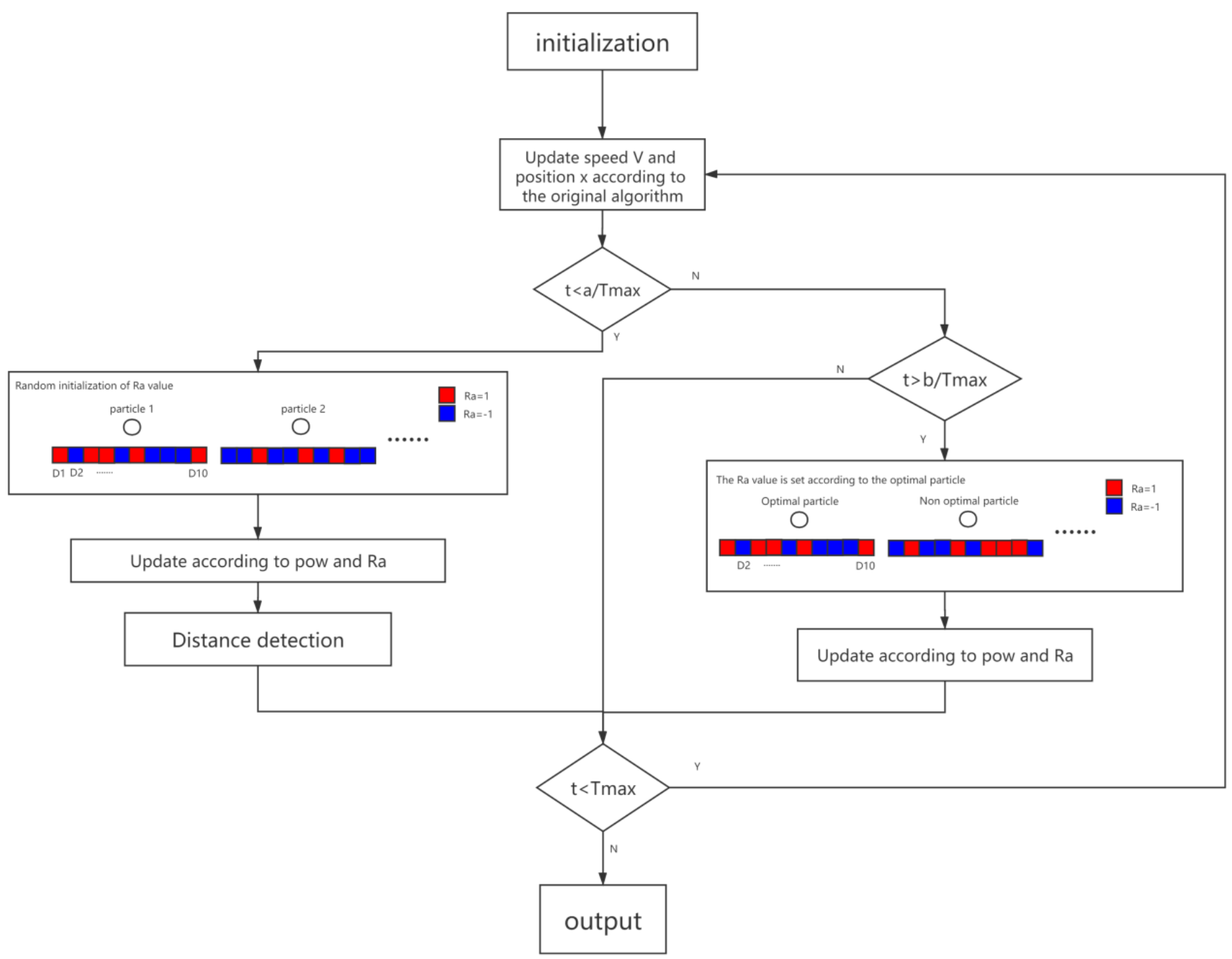

Figure 4. Framework of the RaPSO algorithm.

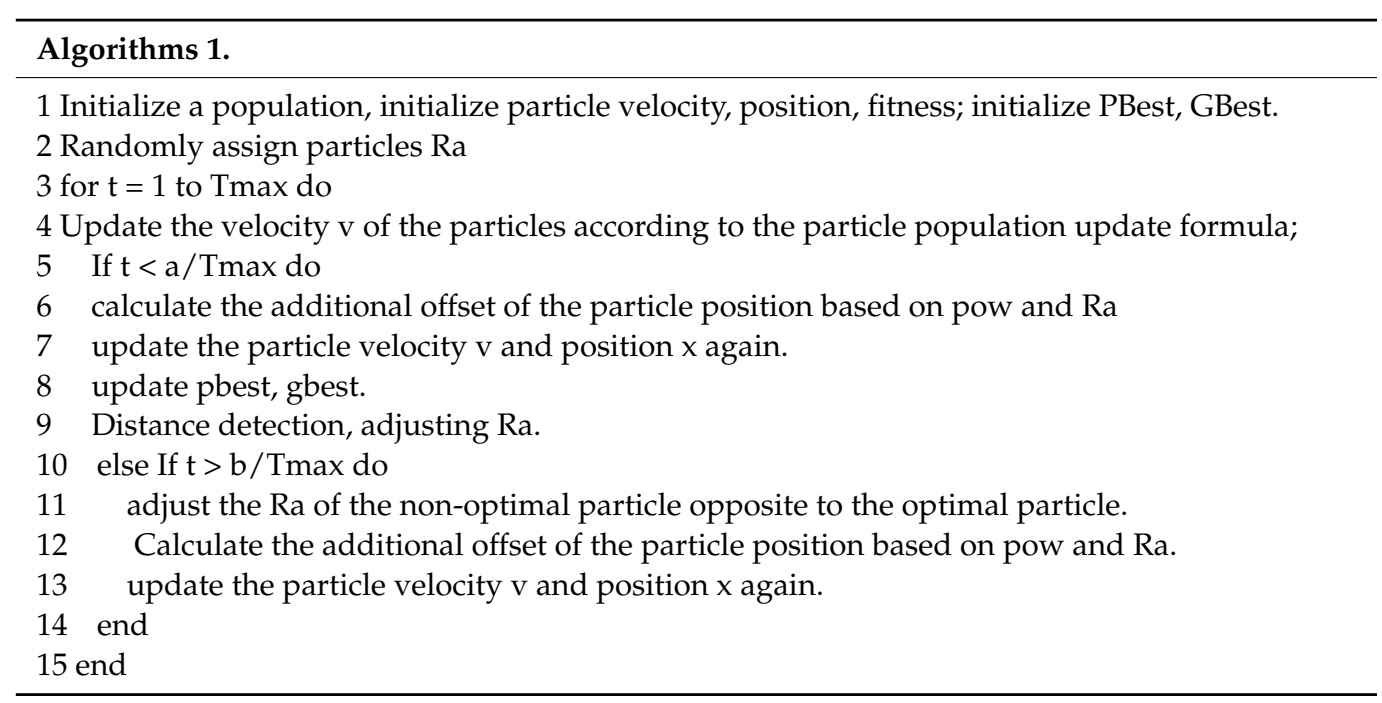




\section{Entropy and Convergence}

The paper discusses the effectiveness of the strategy in terms of entropy and convergence. An increase in population confusion often means a decrease in convergence performance, but if it increases the likelihood that the population searches for the optimal value without affecting convergence, i.e., it produces small fluctuations in the convergence curve, it will help improve the algorithm. We chose population entropy and DBscan as reference tools to draw figures to establish that the multi-stage search strategy worked.

\subsection{Population Entropy}

Entropy is a measure of the degree of chaos in a system and population entropy is an indicator of group diversity. The general definition of entropy may not apply in high-dimensional space; therefore, we chose population entropy as the evaluation criterion of algorithm convergence. In this paper, we assume that the number of particles is $M$, and the best individual position of the particles is $\mathrm{f}_{-} \mathrm{pb}$. Calculating population entropy can be divided into three steps:

Step 1: Calculate the minimum fitness of the particle's historical best position, $\mathrm{f}_{\min }=\min \left(\mathrm{f}_{-} \mathrm{pb}\right)$, and maximum fitness, $\mathrm{f}_{\max }=\max \left(\mathrm{f}_{-} \mathrm{pb}\right)$. The interval of consideration is $\left[\mathrm{f}_{\min }, \mathrm{f}_{\max }\right]$;

Step 2: Divide the interval $\left[\mathrm{f}_{\min }, \mathrm{f}_{\max }\right]$ into $M$ equidistant small regions and calculate the number of $\mathrm{f}_{-} \mathrm{pb}$ in each small interval, $\mathrm{k}_{\mathrm{i}}, \mathrm{i}=1,2, \ldots, \mathrm{M}$, and $\sum_{\mathrm{i}=1}^{\mathrm{M}} \mathrm{k}_{\mathrm{i}}=\mathrm{M}$;

Step 3: Calculate the population entropy, $\mathrm{E}_{\mathrm{t}}=-\sum_{\mathrm{i}=1}^{\mathrm{M}} \mathrm{p}_{\mathrm{i}} \log \mathrm{p}_{\mathrm{i}}, \mathrm{p}_{\mathrm{i}}=\frac{\mathrm{k}_{\mathrm{i}}}{\mathrm{M}}$.

The larger the computational results of population entropy, the more chaotic the particle distribution in space and the less convergent the algorithm.

We compared PSO and the improved algorithm RaPSO using CEC2017-fun10 as the objective function and by conducting experiments in 10-dimensional space. The fitness value of Figure $5 a$ is higher than that of Figure $5 b$. As can be seen from Figure $5 a$, the population entropy value of PSO basically has a continuous decreasing trend, and PSO has nearly no effective exploration in the middle and at the end of the algorithm iteration, after which the fitness value no longer changes. This also demonstrates that PSO is easily trapped in local optima. Figure $5 \mathrm{~b}$ shows the historical change in the population entropy after adding the stage search strategy, from which we can see that the algorithm is in a more chaotic state from beginning to end, and the population entropy is obviously larger than that of PSO at each moment. However, the experimental results are better than those depicted in Figure 5a, which shows that, under the premise of algorithm convergence, increasing population entropy is beneficial to particles in terms of searching for the optimal solution, i.e., increasing population entropy at the beginning of an iteration can help in global exploration, while increasing it at the end of the iteration can benefit local exploitation.

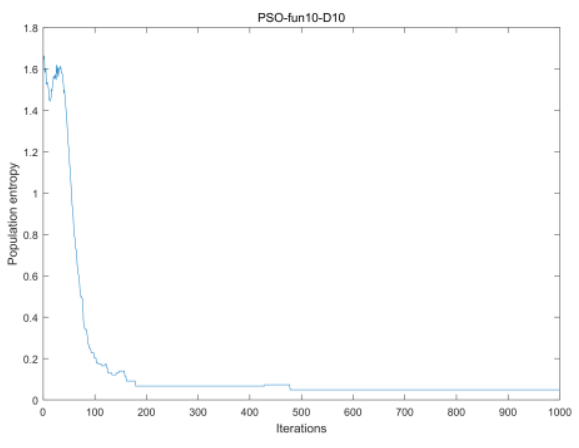

(a)

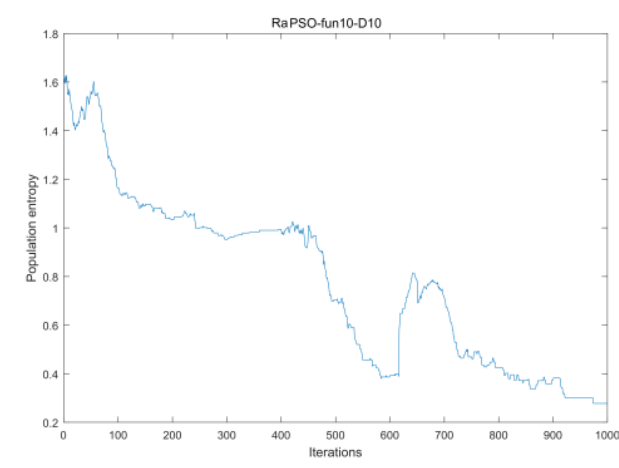

(b)

Figure 5. (a) Population entropy without strategy; (b) population entropy with strategy. 


\subsection{DBscan}

DBscan can measure the degree of population disorder from the perspective of density. It defines clusters as the maximum set of densely connected points, which can divide regions with sufficient density into clusters and find clusters of arbitrary shapes in the spatial database of noise. The DBscan calculation method is described as follows:

Step 1: Define the field radius e and the threshold of core point;

Step 2: Start from one particle and find all particles that have a density connection. Jump out of this cluster and start to find particles again from the other particle;

Step 3: Repeat step 2 until all particles have been traversed.

We chose the conditions for the population entropy experiment and plotted Figure 6. From the figure, we can see that DBscan can depict the clustering phenomenon of the population more graphically than population entropy. The PSO algorithm enters a high degree of clustering after 400 iterations, where the particle population cannot jump out of the local optimum due to insufficient guidance information. Similarly, it can be seen that the overall convergence performance of the population remains unchanged after adding the strategy, while the degree of confusion is improved.

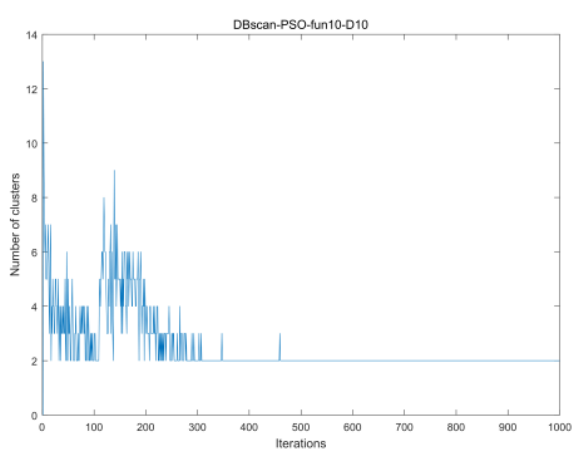

(a)

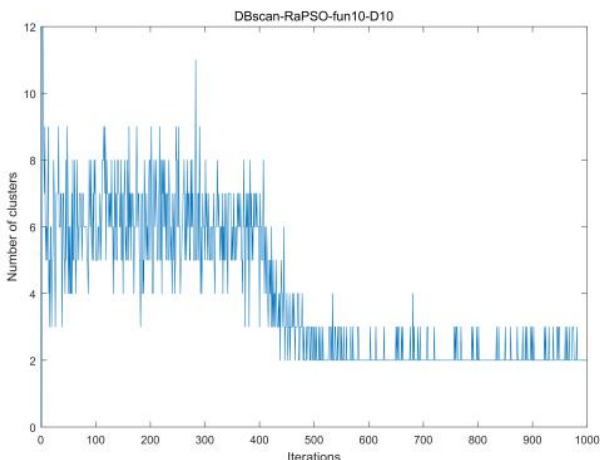

(b)

Figure 6. (a) The number of clusters without applying policies; (b) the number of clusters applying policies.

\section{Experimental Analysis of the Improved Algorithm}

\subsection{Experimental Design Method}

We chose 29 test functions in CEC2017 as objective functions for optimization. Among them, Fun1 is single-peaked, Fun3-Fun10 are multi-peaked, Fun11-Fun20 are mixed, and Fun21-Fun30 are composite. The details are shown in the Table 1.

Table 1. CEC17 functions. U: unimodal; M: multimodal; H: hybrid; C: composition.

\begin{tabular}{|c|c|c|c|}
\hline Num & Function Name & Property & Best Value \\
\hline Fun1 & Shifted and Rotated Bent Cigar Function & $\mathrm{U}$ & 100 \\
\hline Fun3 & Shifted and Rotated Zakharov Function & M & 300 \\
\hline Fun4 & Shifted and Rotated Rosenbrock's Function & M & 400 \\
\hline Fun5 & Shifted and Rotated Rastrigin's Function & M & 500 \\
\hline Fun6 & Shifted and Rotated Expanded Scaffer's F6 Function & M & 600 \\
\hline Fun7 & Shifted and Rotated Lunacek Bi_Rastrigin Function & M & 700 \\
\hline Fun8 & Shifted and Rotated Non-Continuous Rastrigin's Function & M & 800 \\
\hline Fun9 & Shifted and Rotated Levy Function & M & 900 \\
\hline Fun10 & Shifted and Rotated Schwefel's Function & M & 1000 \\
\hline Fun11 & Hybrid Function $1(\mathrm{~N}=3)$ & $\mathrm{H}$ & 1100 \\
\hline Fun12 & Hybrid Function $2(\mathrm{~N}=3)$ & $\mathrm{H}$ & 1200 \\
\hline Fun13 & Hybrid Function $3(\mathrm{~N}=3)$ & $\mathrm{H}$ & 1300 \\
\hline Fun14 & Hybrid Function $4(\mathrm{~N}=4)$ & $\mathrm{H}$ & 1400 \\
\hline Fun15 & Hybrid Function $5(\mathrm{~N}=4)$ & $\mathrm{H}$ & 1500 \\
\hline
\end{tabular}


Table 1. Cont.

\begin{tabular}{cccc}
\hline Num & Function Name & Property & Best Value \\
\hline Fun16 & Hybrid Function 6 $(\mathrm{N}=4)$ & $\mathrm{H}$ & 1600 \\
Fun17 & Hybrid Function 6 $(\mathrm{N}=5)$ & $\mathrm{H}$ & $\mathrm{H}$ \\
Fun18 & Hybrid Function 6 $(\mathrm{N}=5)$ & $\mathrm{H}$ & 1700 \\
Fun19 & Hybrid Function 6 $(\mathrm{N}=5)$ & $\mathrm{H}$ & 1800 \\
Fun20 & Hybrid Function 6 $(\mathrm{N}=6)$ & $\mathrm{C}$ & 2000 \\
Fun21 & Composition Function 1 $(\mathrm{N}=3)$ & $\mathrm{C}$ & 2100 \\
Fun22 & Composition Function 2 $(\mathrm{N}=3)$ & $\mathrm{C}$ & 2200 \\
Fun23 & Composition Function 3 $(\mathrm{N}=4)$ & $\mathrm{C}$ & 2300 \\
Fun24 & Composition Function 4 $(\mathrm{N}=4)$ & $\mathrm{C}$ & 2400 \\
Fun25 & Composition Function 5 $(\mathrm{N}=5)$ & $\mathrm{C}$ & 2500 \\
Fun26 & Composition Function 6 $(\mathrm{N}=5)$ & $\mathrm{C}$ & 2600 \\
Fun27 & Composition Function 7 $(\mathrm{N}=6)$ & $\mathrm{C}$ & 2700 \\
Fun28 & Composition Function 8 $(\mathrm{N}=6)$ & $\mathrm{C}$ & 2800 \\
Fun29 & Composition Function 9 $(\mathrm{N}=3)$ & $\mathrm{C}$ & 2900 \\
Fun30 & Composition Function 10 $(\mathrm{N}=3)$ & & 3000 \\
\hline
\end{tabular}

\subsection{Experimental Parameter Selection}

The 29 test functions of CEC2017 were selected as target functions for optimization tests, and experiments were conducted with a population size $\mathrm{N}=100$, repeat $=30$, and $\mathrm{D}=10,30$. Other parameter settings are shown in Table 2. In order to achieve a better comparison, the parameters were all selected based on previous experiments [24].

Table 2. Parameter selection of the three algorithms.

$$
\begin{gathered}
\mathrm{w}=0.5, \mathrm{c}_{1}=\mathrm{c}_{2}=2 \\
\mathrm{w}=0.9-0.4, \mathrm{c}_{1}=\mathrm{c}_{2}=1.49445, \mathrm{c}_{3}=0.5-2.5 \\
\mathrm{w}=0.99-0.2, \mathrm{c}_{1}=2.5-0.5, \mathrm{c}_{2}=0.5-2.5, \mathrm{c}=3-1.5, \mathrm{~g}_{1}=37, \mathrm{~g}_{2}=63
\end{gathered}
$$

After adding the improvement mechanism, there were three additional controllable parameters in each algorithm: str, prop, and stage. In order to ensure the accuracy of parameter selection, the number of experimental repetitions was increased to 100 .

\subsubsection{The Parameter Str}

The parameter str affects the magnitude of the strength of all repulsions and attractions in stages 1 and 3. The algorithm used in the experiment was the standard PSO. With the other parameters unchanged, Fun5, Fun14, and Fun23 in CEC2017 were selected as objects for the comparison experiments. The $x$-axis in Figure 7 represents the value of parameter str, and the $y$-axis represents the average fitness after normalization. The value range of the $x$-axis was obtained after a large number of experiments. The experimental range was far from that. It was the range obtained after preliminary screening. The sub-graph in the Figure 7 is a detailed display of the $x$-axis from 20 to 30 . The results from Figure 7 show that $\operatorname{str}=25$ was chosen to obtain better results.

\subsubsection{The Parameter Prop}

The parameter prop determines the proportion of poorly adapted particles in stage 1. Because only Fun5 in CEC2017 was selected as the test function, the fitness value of the $y$-axis was unprocessed. Other parts are similar to those used in the experiment in Section 5.2.1 Based on the experimental results, a value of 0.5 was chosen in Figure 8, meaning that half of the particles were attracted to or repelled by the other half. 


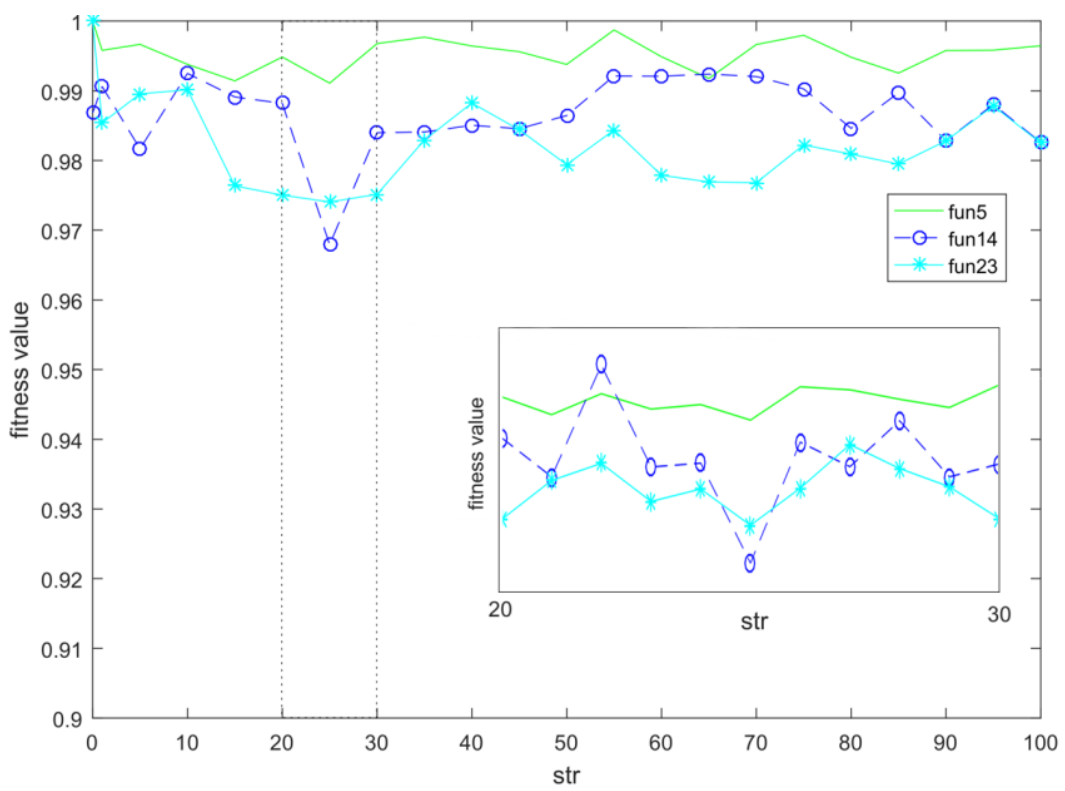

Figure 7. Str parameter selection.

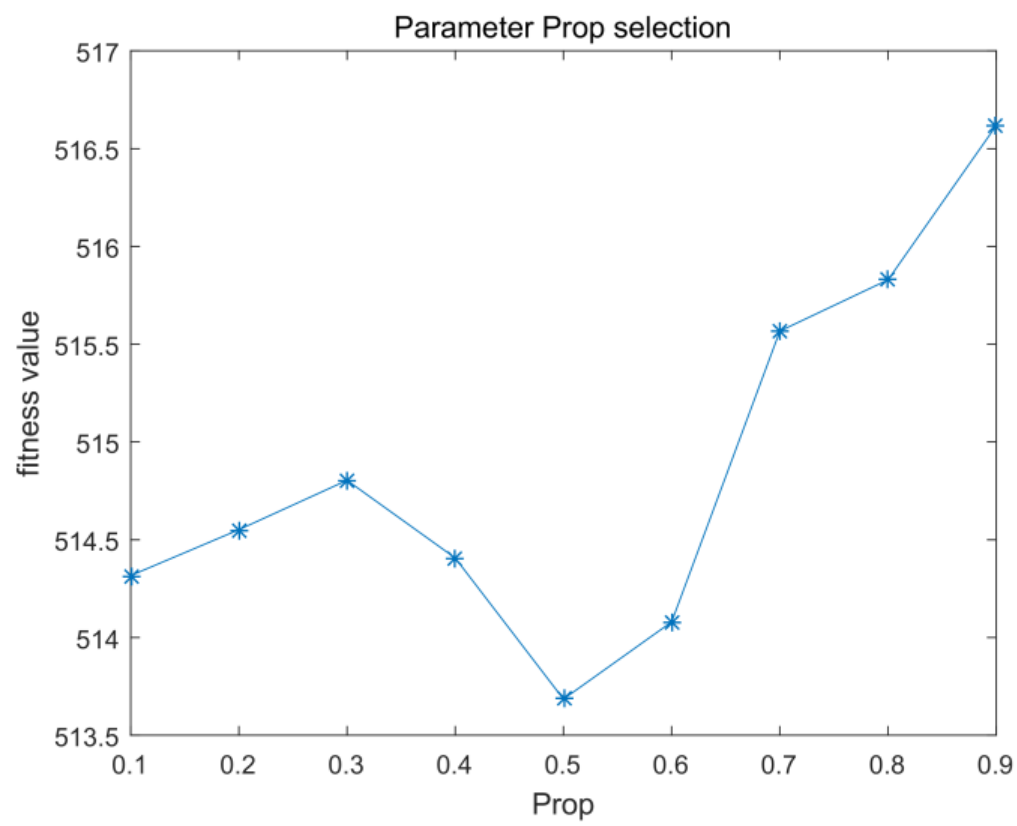

Figure 8. Prop parameter selection.

\subsubsection{The Parameter Stage}

The parameter stage is the basis for deciding the division of the three stages. Similarly, we used standard PSO as the experimental algorithm in 10 dimensions. The test function was Fun25 in CEC2017. Based on the experimental results in Table 3, this was set to 0.1, which means that the first stage accounts for $40 \%$ of the iterations, the second stage $20 \%$, and the third stage $40 \%$. 
Table 3. Stage parameter selection.

\begin{tabular}{ccccc}
\hline Stage Value & Min & Max & Ave & Std \\
\hline 0 & 2893.39 & 2954.96 & 2931.93 & 22.69 \\
0.05 & 2893.58 & 2956.03 & 2928.58 & 23.68 \\
0.1 & 2892.97 & 2957.38 & 2927.66 & 25.22 \\
0.15 & 2893.77 & 2957.98 & 2934.97 & 22.25 \\
0.2 & 2892.86 & 2959.27 & 2935.50 & 21.88 \\
0.25 & 2892.85 & 2953.28 & 2934.69 & 20.67 \\
0.3 & 2893.24 & 2960.55 & 2933.29 & 22.98 \\
0.35 & 2893.69 & 2956.03 & 2932.67 & 22.84 \\
0.4 & 2892.68 & 2955.04 & 2931.57 & 23.60 \\
0.45 & 2893.62 & 2959.70 & 2932.11 & 22.51 \\
0.5 & 2893.16 & 2953.78 & 2934.50 & 21.79 \\
\hline
\end{tabular}

\subsection{Analysis of Experimental Results}

\subsubsection{Standard PSO Based on Multi-Stage Search Strategy}

Tables 4 and 5 is the experiment data of results of PSO and RaPSO in the case of 10/30 dimensions according to the parameters mentioned in Section 5.2. The data in bold in the tables is preferred.

Table 4. Comparison of experimental results of PSO and RaPSO in the case of 10 dimensions.

\begin{tabular}{|c|c|c|c|c|c|c|c|c|}
\hline \multirow{2}{*}{ Fun } & \multicolumn{4}{|c|}{ PSO (Dim = 10) } & \multicolumn{4}{|c|}{ RaPSO (Dim = 10) } \\
\hline & Min & Max & Mean & Std & Min & Max & Mean & Std \\
\hline $\mathrm{F} 1$ & $1.02 \times 10^{2}$ & $2.54 \times 10^{3}$ & $1.29 \times 10^{3}$ & $8.30 \times 10^{2}$ & $1.13 \times 10^{2}$ & $1.53 \times 10^{3}$ & $7.13 \times 10^{2}$ & $5.06 \times 10^{2}$ \\
\hline F3 & $3.00 \times 10^{2}$ & $3.00 \times 10^{2}$ & $3.00 \times 10^{2}$ & $1.06 \times 10^{-14}$ & $3.00 \times 10^{2}$ & $3.00 \times 10^{2}$ & $3.00 \times 10^{2}$ & $9.64 \times 10^{-11}$ \\
\hline F4 & $4.00 \times 10^{2}$ & $4.35 \times 10^{2}$ & $4.17 \times 10^{2}$ & $1.66 \times 10^{1}$ & $4.00 \times 10^{2}$ & $4.35 \times 10^{2}$ & $4.16 \times 10^{2}$ & $1.66 \times 10^{1}$ \\
\hline F5 & $5.04 \times 10^{2}$ & $5.34 \times 10^{2}$ & $5.16 \times 10^{2}$ & $7.92 \times 10^{0}$ & $5.04 \times 10^{2}$ & $5.18 \times 10^{2}$ & $5.11 \times 10^{2}$ & $3.53 \times 10^{0}$ \\
\hline F6 & $6.00 \times 10^{2}$ & $6.00 \times 10^{2}$ & $6.00 \times 10^{2}$ & $4.22 \times 10^{-14}$ & $6.00 \times 10^{2}$ & $6.00 \times 10^{2}$ & $6.00 \times 10^{2}$ & $3.69 \times 10^{-13}$ \\
\hline F7 & $7.13 \times 10^{2}$ & $7.28 \times 10^{2}$ & $7.20 \times 10^{2}$ & $3.77 \times 10^{0}$ & $7.14 \times 10^{2}$ & $7.22 \times 10^{2}$ & $7.18 \times 10^{2}$ & $2.29 \times 10^{0}$ \\
\hline F8 & $8.10 \times 10^{2}$ & $8.40 \times 10^{2}$ & $8.21 \times 10^{2}$ & $7.84 \times 10^{0}$ & $8.06 \times 10^{2}$ & $8.19 \times 10^{2}$ & $8.13 \times 10^{2}$ & $3.22 \times 10^{0}$ \\
\hline F9 & $9.00 \times 10^{2}$ & $9.00 \times 10^{2}$ & $9.00 \times 10^{2}$ & $0.00 \times 10^{0}$ & $9.00 \times 10^{2}$ & $9.00 \times 10^{2}$ & $9.00 \times 10^{2}$ & $0.00 \times 10^{0}$ \\
\hline F10 & $1.13 \times 10^{3}$ & $1.85 \times 10^{3}$ & $1.42 \times 10^{3}$ & $1.93 \times 10^{2}$ & $1.01 \times 10^{3}$ & $1.51 \times 10^{3}$ & $1.30 \times 10^{3}$ & $1.38 \times 10^{2}$ \\
\hline F11 & $1.10 \times 10^{3}$ & $1.13 \times 10^{3}$ & $1.11 \times 10^{3}$ & $7.65 \times 10^{0}$ & $1.10 \times 10^{3}$ & $1.12 \times 10^{3}$ & $1.11 \times 10^{3}$ & $4.54 \times 10^{0}$ \\
\hline F12 & $1.50 \times 10^{3}$ & $4.31 \times 10^{5}$ & $2.66 \times 10^{4}$ & $7.69 \times 10^{4}$ & $1.72 \times 10^{3}$ & $2.37 \times 10^{4}$ & $1.13 \times 10^{4}$ & $6.96 \times 10^{3}$ \\
\hline F13 & $1.31 \times 10^{3}$ & $8.14 \times 10^{3}$ & $3.09 \times 10^{3}$ & $2.00 \times 10^{3}$ & $1.31 \times 10^{3}$ & $4.62 \times 10^{3}$ & $2.33 \times 10^{3}$ & $9.75 \times 10^{2}$ \\
\hline F14 & $1.44 \times 10^{3}$ & $1.56 \times 10^{3}$ & $1.48 \times 10^{3}$ & $2.63 \times 10^{1}$ & $1.44 \times 10^{3}$ & $1.49 \times 10^{3}$ & $1.47 \times 10^{3}$ & $1.32 \times 10^{1}$ \\
\hline F15 & $1.51 \times 10^{3}$ & $1.73 \times 10^{3}$ & $1.56 \times 10^{3}$ & $5.09 \times 10^{1}$ & $1.50 \times 10^{3}$ & $1.58 \times 10^{3}$ & $1.54 \times 10^{3}$ & $1.96 \times 10^{1}$ \\
\hline F16 & $1.60 \times 10^{3}$ & $1.85 \times 10^{3}$ & $1.71 \times 10^{3}$ & $7.88 \times 10^{1}$ & $1.60 \times 10^{3}$ & $1.73 \times 10^{3}$ & $1.69 \times 10^{3}$ & $5.15 \times 10^{1}$ \\
\hline F17 & $1.72 \times 10^{3}$ & $1.81 \times 10^{3}$ & $1.75 \times 10^{3}$ & $1.94 \times 10^{1}$ & $1.73 \times 10^{3}$ & $1.75 \times 10^{3}$ & $1.74 \times 10^{3}$ & $8.12 \times 10^{0}$ \\
\hline F18 & $1.87 \times 10^{3}$ & $2.15 \times 10^{4}$ & $7.02 \times 10^{3}$ & $4.88 \times 10^{3}$ & $2.00 \times 10^{3}$ & $1.12 \times 10^{4}$ & $6.13 \times 10^{3}$ & $2.85 \times 10^{3}$ \\
\hline F19 & $1.90 \times 10^{3}$ & $2.00 \times 10^{3}$ & $1.92 \times 10^{3}$ & $2.22 \times 10^{1}$ & $1.91 \times 10^{3}$ & $1.94 \times 10^{3}$ & $1.92 \times 10^{3}$ & $8.57 \times 10^{0}$ \\
\hline F20 & $2.01 \times 10^{3}$ & $2.20 \times 10^{3}$ & $2.07 \times 10^{3}$ & $5.13 \times 10^{1}$ & $2.01 \times 10^{3}$ & $2.09 \times 10^{3}$ & $2.04 \times 10^{3}$ & $1.95 \times 10^{1}$ \\
\hline F21 & $2.25 \times 10^{3}$ & $2.25 \times 10^{3}$ & $2.25 \times 10^{3}$ & $8.44 \times 10^{-14}$ & $2.20 \times 10^{3}$ & $2.20 \times 10^{3}$ & $2.20 \times 10^{3}$ & $1.19 \times 10^{-13}$ \\
\hline $\mathrm{F} 22$ & $2.21 \times 10^{3}$ & $2.35 \times 10^{3}$ & $2.35 \times 10^{3}$ & $2.56 \times 10^{1}$ & $2.20 \times 10^{3}$ & $2.30 \times 10^{3}$ & $2.30 \times 10^{3}$ & $1.83 \times 10^{1}$ \\
\hline F23 & $2.40 \times 10^{3}$ & $2.83 \times 10^{3}$ & $2.67 \times 10^{3}$ & $1.18 \times 10^{2}$ & $2.40 \times 10^{3}$ & $2.69 \times 10^{3}$ & $2.63 \times 10^{3}$ & $9.40 \times 10^{1}$ \\
\hline F24 & $2.50 \times 10^{3}$ & $2.83 \times 10^{3}$ & $2.62 \times 10^{3}$ & $9.09 \times 10^{1}$ & $2.50 \times 10^{3}$ & $2.60 \times 10^{3}$ & $2.58 \times 10^{3}$ & $3.79 \times 10^{1}$ \\
\hline F25 & $2.89 \times 10^{3}$ & $2.96 \times 10^{3}$ & $2.92 \times 10^{3}$ & $2.55 \times 10^{1}$ & $2.90 \times 10^{3}$ & $2.95 \times 10^{3}$ & $2.93 \times 10^{3}$ & $2.15 \times 10^{1}$ \\
\hline F26 & $2.80 \times 10^{3}$ & $3.76 \times 10^{3}$ & $2.94 \times 10^{3}$ & $2.67 \times 10^{2}$ & $2.60 \times 10^{3}$ & $2.90 \times 10^{3}$ & $2.84 \times 10^{3}$ & $8.14 \times 10^{1}$ \\
\hline $\mathrm{F} 27$ & $3.15 \times 10^{3}$ & $3.46 \times 10^{3}$ & $3.27 \times 10^{3}$ & $8.58 \times 10^{1}$ & $3.10 \times 10^{3}$ & $3.28 \times 10^{3}$ & $3.18 \times 10^{3}$ & $5.27 \times 10^{1}$ \\
\hline F28 & $3.10 \times 10^{3}$ & $3.15 \times 10^{3}$ & $3.14 \times 10^{3}$ & $2.21 \times 10^{1}$ & $3.10 \times 10^{3}$ & $3.15 \times 10^{3}$ & $3.13 \times 10^{3}$ & $2.35 \times 10^{1}$ \\
\hline F29 & $3.14 \times 10^{3}$ & $3.30 \times 10^{3}$ & $3.18 \times 10^{3}$ & $3.60 \times 10^{1}$ & $3.14 \times 10^{3}$ & $3.20 \times 10^{3}$ & $3.17 \times 10^{3}$ & $1.45 \times 10^{1}$ \\
\hline F30 & $3.50 \times 10^{3}$ & $3.70 \times 10^{4}$ & $1.15 \times 10^{4}$ & $9.46 \times 10^{3}$ & $3.43 \times 10^{3}$ & $1.04 \times 10^{4}$ & $5.61 \times 10^{3}$ & $1.91 \times 10^{3}$ \\
\hline
\end{tabular}


Table 5. Comparison of experimental results of PSO and RaPSO in the case of 30 dimensions.

\begin{tabular}{|c|c|c|c|c|c|c|c|c|}
\hline \multirow{2}{*}{ Fun } & \multicolumn{4}{|c|}{ PSO (Dim = 30) } & \multicolumn{4}{|c|}{ RaPSO (Dim = 30) } \\
\hline & Min & Max & Mean & Std & Min & Max & Mean & Std \\
\hline $\mathrm{F} 1$ & $1.00 \times 10^{2}$ & $1.07 \times 10^{9}$ & $1.02 \times 10^{8}$ & $3.13 \times 10^{8}$ & $1.00 \times 10^{2}$ & $1.91 \times 10^{3}$ & $2.42 \times 10^{2}$ & $3.72 \times 10^{2}$ \\
\hline F3 & $3.02 \times 10^{2}$ & $4.20 \times 10^{2}$ & $3.27 \times 10^{2}$ & $2.60 \times 10^{1}$ & $4.03 \times 10^{2}$ & $5.69 \times 10^{2}$ & $4.81 \times 10^{2}$ & $5.06 \times 10^{1}$ \\
\hline $\mathrm{F} 4$ & $4.04 \times 10^{2}$ & $5.80 \times 10^{2}$ & $4.86 \times 10^{2}$ & $4.48 \times 10^{1}$ & $4.04 \times 10^{2}$ & $4.96 \times 10^{2}$ & $4.70 \times 10^{2}$ & $2.00 \times 10^{1}$ \\
\hline F5 & $5.66 \times 10^{2}$ & $6.57 \times 10^{2}$ & $6.05 \times 10^{2}$ & $2.48 \times 10^{1}$ & $5.58 \times 10^{2}$ & $6.04 \times 10^{2}$ & $5.88 \times 10^{2}$ & $1.24 \times 10^{1}$ \\
\hline F6 & $6.00 \times 10^{2}$ & $6.25 \times 10^{2}$ & $6.07 \times 10^{2}$ & $6.46 \times 10^{0}$ & $6.00 \times 10^{2}$ & $6.08 \times 10^{2}$ & $6.04 \times 10^{2}$ & $2.43 \times 10^{0}$ \\
\hline F7 & $7.75 \times 10^{2}$ & $9.14 \times 10^{2}$ & $8.21 \times 10^{2}$ & $2.96 \times 10^{1}$ & $7.70 \times 10^{2}$ & $8.29 \times 10^{2}$ & $8.00 \times 10^{2}$ & $1.74 \times 10^{1}$ \\
\hline F8 & $8.83 \times 10^{2}$ & $9.94 \times 10^{2}$ & $9.21 \times 10^{2}$ & $3.13 \times 10^{1}$ & $8.67 \times 10^{2}$ & $9.34 \times 10^{2}$ & $9.10 \times 10^{2}$ & $1.74 \times 10^{1}$ \\
\hline F9 & $1.01 \times 10^{3}$ & $5.26 \times 10^{3}$ & $2.83 \times 10^{3}$ & $1.16 \times 10^{3}$ & $9.02 \times 10^{2}$ & $2.81 \times 10^{3}$ & $1.94 \times 10^{3}$ & $6.10 \times 10^{2}$ \\
\hline F10 & $2.86 \times 10^{3}$ & $4.84 \times 10^{3}$ & $3.89 \times 10^{3}$ & $5.55 \times 10^{2}$ & $2.60 \times 10^{3}$ & $4.47 \times 10^{3}$ & $3.78 \times 10^{3}$ & $5.39 \times 10^{2}$ \\
\hline F11 & $1.17 \times 10^{3}$ & $1.38 \times 10^{3}$ & $1.24 \times 10^{3}$ & $4.55 \times 10^{1}$ & $1.16 \times 10^{3}$ & $1.28 \times 10^{3}$ & $1.23 \times 10^{3}$ & $3.38 \times 10^{1}$ \\
\hline F12 & $3.02 \times 10^{3}$ & $1.80 \times 10^{7}$ & $1.16 \times 10^{6}$ & $4.33 \times 10^{6}$ & $2.41 \times 10^{3}$ & $1.51 \times 10^{4}$ & $6.47 \times 10^{3}$ & $3.37 \times 10^{3}$ \\
\hline F13 & $1.33 \times 10^{3}$ & $2.36 \times 10^{4}$ & $2.73 \times 10^{3}$ & $4.05 \times 10^{3}$ & $1.36 \times 10^{3}$ & $2.62 \times 10^{3}$ & $1.77 \times 10^{3}$ & $3.73 \times 10^{2}$ \\
\hline F14 & $1.52 \times 10^{3}$ & $1.96 \times 10^{3}$ & $1.67 \times 10^{3}$ & $1.06 \times 10^{2}$ & $1.56 \times 10^{3}$ & $1.76 \times 10^{3}$ & $1.65 \times 10^{3}$ & $6.13 \times 10^{1}$ \\
\hline F15 & $1.53 \times 10^{3}$ & $1.98 \times 10^{3}$ & $1.60 \times 10^{3}$ & $7.78 \times 10^{1}$ & $1.54 \times 10^{3}$ & $1.62 \times 10^{3}$ & $1.58 \times 10^{3}$ & $2.20 \times 10^{1}$ \\
\hline F16 & $2.11 \times 10^{3}$ & $2.98 \times 10^{3}$ & $2.54 \times 10^{3}$ & $2.28 \times 10^{2}$ & $1.76 \times 10^{3}$ & $2.40 \times 10^{3}$ & $2.11 \times 10^{3}$ & $1.77 \times 10^{2}$ \\
\hline F17 & $1.78 \times 10^{3}$ & $2.69 \times 10^{3}$ & $2.12 \times 10^{3}$ & $2.01 \times 10^{2}$ & $1.79 \times 10^{3}$ & $2.09 \times 10^{3}$ & $1.95 \times 10^{3}$ & $8.23 \times 10^{1}$ \\
\hline F18 & $1.01 \times 10^{4}$ & $1.38 \times 10^{5}$ & $5.53 \times 10^{4}$ & $3.32 \times 10^{4}$ & $1.12 \times 10^{4}$ & $8.45 \times 10^{4}$ & $3.96 \times 10^{4}$ & $1.92 \times 10^{4}$ \\
\hline F19 & $2.01 \times 10^{3}$ & $1.64 \times 10^{4}$ & $5.69 \times 10^{3}$ & $3.76 \times 10^{3}$ & $1.94 \times 10^{3}$ & $5.62 \times 10^{3}$ & $3.06 \times 10^{3}$ & $1.01 \times 10^{3}$ \\
\hline F20 & $2.26 \times 10^{3}$ & $2.74 \times 10^{3}$ & $2.47 \times 10^{3}$ & $1.37 \times 10^{2}$ & $2.26 \times 10^{3}$ & $2.56 \times 10^{3}$ & $2.42 \times 10^{3}$ & $7.98 \times 10^{1}$ \\
\hline F21 & $2.20 \times 10^{3}$ & $2.20 \times 10^{3}$ & $2.20 \times 10^{3}$ & $4.78 \times 10^{-13}$ & $2.25 \times 10^{3}$ & $2.25 \times 10^{3}$ & $2.25 \times 10^{3}$ & $5.00 \times 10^{-13}$ \\
\hline $\mathrm{F} 22$ & $2.30 \times 10^{3}$ & $2.30 \times 10^{3}$ & $2.30 \times 10^{3}$ & $4.55 \times 10^{-13}$ & $2.35 \times 10^{3}$ & $2.35 \times 10^{3}$ & $2.35 \times 10^{3}$ & $4.55 \times 10^{-13}$ \\
\hline F23 & $2.96 \times 10^{3}$ & $4.50 \times 10^{3}$ & $3.53 \times 10^{3}$ & $3.57 \times 10^{2}$ & $2.98 \times 10^{3}$ & $4.01 \times 10^{3}$ & $3.46 \times 10^{3}$ & $3.14 \times 10^{2}$ \\
\hline $\mathrm{F} 24$ & $2.60 \times 10^{3}$ & $2.61 \times 10^{3}$ & $2.60 \times 10^{3}$ & $1.62 \times 10^{0}$ & $2.60 \times 10^{3}$ & $2.60 \times 10^{3}$ & $2.60 \times 10^{3}$ & $1.16 \times 10^{-12}$ \\
\hline F25 & $2.90 \times 10^{3}$ & $3.12 \times 10^{3}$ & $2.97 \times 10^{3}$ & $5.84 \times 10^{1}$ & $2.90 \times 10^{3}$ & $2.97 \times 10^{3}$ & $2.93 \times 10^{3}$ & $3.08 \times 101$ \\
\hline F26 & $2.80 \times 10^{3}$ & $5.43 \times 10^{3}$ & $2.90 \times 10^{3}$ & $4.79 \times 10^{2}$ & $2.80 \times 10^{3}$ & $2.80 \times 10^{3}$ & $2.80 \times 10^{3}$ & $1.50 \times 10^{-12}$ \\
\hline $\mathrm{F} 27$ & $3.81 \times 10^{3}$ & $5.30 \times 10^{3}$ & $4.53 \times 10^{3}$ & $3.39 \times 10^{2}$ & $3.73 \times 10^{3}$ & $4.47 \times 10^{3}$ & $4.17 \times 10^{3}$ & $2.25 \times 10^{2}$ \\
\hline F28 & $3.22 \times 10^{3}$ & $3.42 \times 10^{3}$ & $3.30 \times 10^{3}$ & $5.42 \times 10^{1}$ & $3.22 \times 10^{3}$ & $3.30 \times 10^{3}$ & $3.26 \times 10^{3}$ & $2.43 \times 10^{1}$ \\
\hline F29 & $3.31 \times 10^{3}$ & $4.10 \times 10^{3}$ & $3.69 \times 10^{3}$ & $2.25 \times 10^{2}$ & $3.28 \times 10^{3}$ & $3.63 \times 10^{3}$ & $3.44 \times 10^{3}$ & $8.41 \times 10^{1}$ \\
\hline F30 & $4.23 \times 10^{3}$ & $5.44 \times 10^{4}$ & $9.02 \times 10^{3}$ & $9.30 \times 10^{3}$ & $4.09 \times 10^{3}$ & $1.43 \times 10^{4}$ & $6.76 \times 10^{3}$ & $2.78 \times 10^{3}$ \\
\hline
\end{tabular}

\subsubsection{TSLPSO Based on Multi-Stage Search Strategy}

Tables 6 and 7 is the experiment data of results of TSLPSO and RaTSLPSO in the case of 10/30 dimensions according to the parameters mentioned in Section 5.2. The data in bold in the tables is preferred.

Table 6. Comparison of experimental results of TSLPSO and RaTSLPSO in the case of 10 dimensions.

\begin{tabular}{|c|c|c|c|c|c|c|c|c|}
\hline \multirow{2}{*}{ Fun } & \multicolumn{4}{|c|}{ TSLPSO $($ Dim $=10)$} & \multicolumn{4}{|c|}{ RaTSLPSO (Dim = 10) } \\
\hline & Min & Max & Mean & Std & Min & $\operatorname{Max}$ & Mean & Std \\
\hline $\mathrm{F} 1$ & $1.31 \times 10^{2}$ & $1.43 \times 10^{3}$ & $7.12 \times 10^{2}$ & $3.85 \times 10^{2}$ & $1.00 \times 10^{2}$ & $1.46 \times 10^{3}$ & $6.30 \times 10^{2}$ & $4.44 \times 10^{2}$ \\
\hline F3 & $3.00 \times 10^{2}$ & $3.00 \times 10^{2}$ & $3.00 \times 10^{2}$ & $1.26 \times 10^{-5}$ & $3.00 \times 10^{2}$ & $3.00 \times 10^{2}$ & $3.00 \times 10^{2}$ & $2.22 \times 10^{-3}$ \\
\hline $\mathrm{F} 4$ & $4.00 \times 10^{2}$ & $4.05 \times 10^{2}$ & $4.01 \times 10^{2}$ & $1.40 \times 10^{0}$ & $4.00 \times 10^{2}$ & $4.05 \times 10^{2}$ & $4.02 \times 10^{2}$ & $1.76 \times 10^{0}$ \\
\hline F5 & $5.03 \times 10^{2}$ & $5.14 \times 10^{2}$ & $5.08 \times 10^{2}$ & $2.56 \times 10^{0}$ & $5.03 \times 10^{2}$ & $5.10 \times 10^{2}$ & $5.07 \times 10^{2}$ & $1.74 \times 10^{0}$ \\
\hline F6 & $6.00 \times 10^{2}$ & $6.00 \times 10^{2}$ & $6.00 \times 10^{2}$ & $2.72 \times 10^{-5}$ & $6.00 \times 10^{2}$ & $6.00 \times 10^{2}$ & $6.00 \times 10^{2}$ & $3.44 \times 10^{-9}$ \\
\hline F7 & $7.12 \times 10^{2}$ & $7.23 \times 10^{2}$ & $7.19 \times 10^{2}$ & $2.82 \times 10^{0}$ & $7.08 \times 10^{2}$ & $7.23 \times 10^{2}$ & $7.19 \times 10^{2}$ & $3.43 \times 10^{0}$ \\
\hline F8 & $8.04 \times 10^{2}$ & $8.12 \times 10^{2}$ & $8.08 \times 10^{2}$ & $2.25 \times 10^{0}$ & $8.03 \times 10^{2}$ & $8.10 \times 10^{2}$ & $8.07 \times 10^{2}$ & $1.79 \times 10^{0}$ \\
\hline F9 & $9.00 \times 10^{2}$ & $9.02 \times 10^{2}$ & $9.00 \times 10^{2}$ & $4.43 \times 10^{-1}$ & $9.00 \times 10^{2}$ & $9.00 \times 10^{2}$ & $9.00 \times 10^{2}$ & $2.73 \times 10^{-2}$ \\
\hline F10 & $1.01 \times 10^{3}$ & $1.40 \times 10^{3}$ & $1.21 \times 10^{3}$ & $1.05 \times 10^{2}$ & $1.00 \times 10^{3}$ & $1.36 \times 10^{3}$ & $1.21 \times 10^{3}$ & $1.16 \times 10^{2}$ \\
\hline F11 & $1.10 \times 10^{3}$ & $1.11 \times 10^{3}$ & $1.10 \times 10^{3}$ & $1.94 \times 10^{0}$ & $1.10 \times 10^{3}$ & $1.11 \times 10^{3}$ & $1.10 \times 10^{3}$ & $1.38 \times 10^{0}$ \\
\hline F12 & $2.41 \times 10^{3}$ & $2.98 \times 10^{4}$ & $1.42 \times 10^{4}$ & $8.42 \times 10^{3}$ & $2.13 \times 10^{3}$ & $2.25 \times 10^{4}$ & $1.13 \times 10^{4}$ & $6.36 \times 10^{3}$ \\
\hline F13 & $1.31 \times 10^{3}$ & $1.46 \times 10^{3}$ & $1.37 \times 10^{3}$ & $3.47 \times 10^{1}$ & $1.31 \times 10^{3}$ & $1.98 \times 10^{3}$ & $1.52 \times 10^{3}$ & $1.99 \times 10^{2}$ \\
\hline F14 & $1.42 \times 10^{3}$ & $1.48 \times 10^{3}$ & $1.44 \times 10^{3}$ & $1.27 \times 10^{1}$ & $1.41 \times 10^{3}$ & $1.44 \times 10^{3}$ & $1.43 \times 10^{3}$ & $6.42 \times 10^{0}$ \\
\hline F15 & $1.50 \times 10^{3}$ & $1.58 \times 10^{3}$ & $1.53 \times 10^{3}$ & $1.91 \times 10^{1}$ & $1.50 \times 10^{3}$ & $1.53 \times 10^{3}$ & $1.52 \times 10^{3}$ & $8.45 \times 10^{0}$ \\
\hline F16 & $1.60 \times 10^{3}$ & $1.62 \times 10^{3}$ & $1.61 \times 10^{3}$ & $4.05 \times 10^{0}$ & $1.60 \times 10^{3}$ & $1.61 \times 10^{3}$ & $1.60 \times 10^{3}$ & $1.85 \times 10^{0}$ \\
\hline F17 & $1.71 \times 10^{3}$ & $1.75 \times 10^{3}$ & $1.73 \times 10^{3}$ & $7.73 \times 10^{0}$ & $1.71 \times 10^{3}$ & $1.74 \times 10^{3}$ & $1.73 \times 10^{3}$ & $6.45 \times 10^{0}$ \\
\hline F18 & $1.85 \times 10^{3}$ & $3.34 \times 10^{3}$ & $2.27 \times 10^{3}$ & $2.99 \times 10^{2}$ & $1.96 \times 10^{3}$ & $2.87 \times 10^{3}$ & $2.38 \times 10^{3}$ & $2.88 \times 10^{2}$ \\
\hline F19 & $1.90 \times 10^{3}$ & $1.92 \times 10^{3}$ & $1.91 \times 10^{3}$ & $3.61 \times 10^{0}$ & $1.90 \times 10^{3}$ & $1.91 \times 10^{3}$ & $1.91 \times 10^{3}$ & $2.47 \times 10^{0}$ \\
\hline F20 & $2.00 \times 10^{3}$ & $2.04 \times 10^{3}$ & $2.02 \times 10^{3}$ & $6.89 \times 10^{0}$ & $2.00 \times 10^{3}$ & $2.03 \times 10^{3}$ & $2.02 \times 10^{3}$ & $9.97 \times 10^{0}$ \\
\hline $\mathrm{F} 21$ & $2.20 \times 10^{3}$ & $2.20 \times 10^{3}$ & $2.20 \times 10^{3}$ & $7.51 \times 10^{-13}$ & $2.20 \times 10^{3}$ & $2.20 \times 10^{3}$ & $2.20 \times 10^{3}$ & $2.14 \times 10^{-7}$ \\
\hline F22 & $2.20 \times 10^{3}$ & $2.30 \times 10^{3}$ & $2.23 \times 10^{3}$ & $3.81 \times 10^{1}$ & $2.20 \times 10^{3}$ & $2.30 \times 10^{3}$ & $2.23 \times 10^{3}$ & $3.80 \times 10^{1}$ \\
\hline F23 & $2.65 \times 10^{3}$ & $2.67 \times 10^{3}$ & $2.66 \times 10^{3}$ & $3.51 \times 10^{0}$ & $2.64 \times 10^{3}$ & $2.66 \times 10^{3}$ & $2.66 \times 10^{3}$ & $5.08 \times 10^{0}$ \\
\hline
\end{tabular}


Table 6. Cont.

\begin{tabular}{|c|c|c|c|c|c|c|c|c|}
\hline \multirow{2}{*}{ Fun } & \multicolumn{4}{|c|}{ TSLPSO $($ Dim $=10)$} & \multicolumn{4}{|c|}{ RaTSLPSO (Dim = 10) } \\
\hline & Min & Max & Mean & Std & Min & Max & Mean & Std \\
\hline F24 & $2.43 \times 10^{3}$ & $2.66 \times 10^{3}$ & $2.53 \times 10^{3}$ & $4.81 \times 10^{1}$ & $2.40 \times 10^{3}$ & $2.54 \times 10^{3}$ & $2.50 \times 10^{3}$ & $2.95 \times 10^{1}$ \\
\hline F25 & $2.85 \times 10^{3}$ & $2.90 \times 10^{3}$ & $2.90 \times 10^{3}$ & $9.15 \times 10^{0}$ & $2.85 \times 10^{3}$ & $2.90 \times 10^{3}$ & $2.90 \times 10^{3}$ & $8.92 \times 10^{0}$ \\
\hline F26 & $2.60 \times 10^{3}$ & $2.95 \times 10^{3}$ & $2.74 \times 10^{3}$ & $1.45 \times 10^{2}$ & $2.60 \times 10^{3}$ & $2.90 \times 10^{3}$ & $2.73 \times 10^{3}$ & $1.38 \times 10^{2}$ \\
\hline F27 & $3.11 \times 10^{3}$ & $3.15 \times 10^{3}$ & $3.13 \times 10^{3}$ & $1.16 \times 10^{1}$ & $3.10 \times 10^{3}$ & $3.15 \times 10^{3}$ & $3.13 \times 10^{3}$ & $1.42 \times 10^{1}$ \\
\hline F28 & $3.10 \times 10^{3}$ & $3.15 \times 10^{3}$ & $3.13 \times 10^{3}$ & $2.22 \times 10^{1}$ & $3.06 \times 10^{3}$ & $3.15 \times 10^{3}$ & $3.12 \times 10^{3}$ & $2.44 \times 10^{1}$ \\
\hline F29 & $3.14 \times 10^{3}$ & $3.19 \times 10^{3}$ & $3.16 \times 10^{3}$ & $1.25 \times 10^{1}$ & $3.10 \times 10^{3}$ & $3.17 \times 10^{3}$ & $3.15 \times 10^{3}$ & $1.66 \times 10^{1}$ \\
\hline F30 & $3.43 \times 10^{3}$ & $2.15 \times 10^{4}$ & $7.91 \times 10^{3}$ & $4.06 \times 10^{3}$ & $3.75 \times 10^{3}$ & $1.04 \times 10^{4}$ & $7.26 \times 10^{3}$ & $2.13 \times 10^{3}$ \\
\hline
\end{tabular}

Table 7. Comparison of experimental results of TSLPSO and RaTSLPSO in the case of 30 dimensions.

\begin{tabular}{|c|c|c|c|c|c|c|c|c|}
\hline \multirow{2}{*}{ Fun } & \multicolumn{4}{|c|}{ TSLPSO $(\operatorname{Dim}=30)$} & \multicolumn{4}{|c|}{ RaTSLPSO (Dim = 30) } \\
\hline & Min & $\operatorname{Max}$ & Mean & Std & Min & $\operatorname{Max}$ & Mean & Std \\
\hline F1 & $1.01 \times 10^{2}$ & $4.46 \times 10^{3}$ & $1.39 \times 10^{3}$ & $1.21 \times 10^{3}$ & $1.03 \times 10^{2}$ & $2.49 \times 10^{3}$ & $1.11 \times 10^{3}$ & $9.16 \times 10^{2}$ \\
\hline F3 & $3.46 \times 10^{2}$ & $1.07 \times 10^{4}$ & $1.78 \times 10^{3}$ & $2.08 \times 10^{3}$ & $3.31 \times 10^{2}$ & $7.38 \times 10^{2}$ & $4.37 \times 10^{2}$ & $1.36 \times 10^{2}$ \\
\hline $\mathrm{F} 4$ & $4.00 \times 10^{2}$ & $5.44 \times 10^{2}$ & $4.47 \times 10^{2}$ & $4.22 \times 10^{1}$ & $4.00 \times 10^{2}$ & $4.70 \times 10^{2}$ & $4.22 \times 10^{2}$ & $3.00 \times 10^{1}$ \\
\hline F5 & $5.36 \times 10^{2}$ & $6.03 \times 10^{2}$ & $5.63 \times 10^{2}$ & $1.54 \times 10^{1}$ & $5.38 \times 10^{2}$ & $5.62 \times 10^{2}$ & $5.53 \times 10^{2}$ & $7.30 \times 10^{0}$ \\
\hline F6 & $6.00 \times 10^{2}$ & $6.00 \times 10^{2}$ & $6.00 \times 10^{2}$ & $2.01 \times 10^{-7}$ & $6.00 \times 10^{2}$ & $6.00 \times 10^{2}$ & $6.00 \times 10^{2}$ & $2.10 \times 10^{-13}$ \\
\hline F7 & $7.70 \times 10^{2}$ & $8.41 \times 10^{2}$ & $7.94 \times 10^{2}$ & $1.41 \times 10^{1}$ & $7.61 \times 10^{2}$ & $7.94 \times 10^{2}$ & $7.84 \times 10^{2}$ & $8.10 \times 10^{0}$ \\
\hline F8 & $8.36 \times 10^{2}$ & $9.03 \times 10^{2}$ & $8.64 \times 10^{2}$ & $1.55 \times 10^{1}$ & $8.36 \times 10^{2}$ & $8.62 \times 10^{2}$ & $8.51 \times 10^{2}$ & $7.44 \times 10^{0}$ \\
\hline F9 & $9.01 \times 10^{2}$ & $2.09 \times 10^{3}$ & $1.05 \times 10^{3}$ & $2.46 \times 10^{2}$ & $9.01 \times 10^{2}$ & $9.95 \times 10^{2}$ & $9.45 \times 10^{2}$ & $3.26 \times 10^{1}$ \\
\hline F10 & $1.97 \times 10^{3}$ & $4.42 \times 10^{3}$ & $3.23 \times 10^{3}$ & $6.23 \times 10^{2}$ & $2.29 \times 10^{3}$ & $3.32 \times 10^{3}$ & $2.85 \times 10^{3}$ & $2.88 \times 10^{2}$ \\
\hline F11 & $1.12 \times 10^{3}$ & $1.30 \times 10^{3}$ & $1.18 \times 10^{3}$ & $4.69 \times 10^{1}$ & $1.13 \times 10^{3}$ & $1.16 \times 10^{3}$ & $1.15 \times 10^{3}$ & $1.20 \times 10^{1}$ \\
\hline F12 & $2.45 \times 10^{3}$ & $1.86 \times 10^{4}$ & $7.36 \times 10^{3}$ & $4.16 \times 10^{3}$ & $2.78 \times 10^{3}$ & $6.16 \times 10^{3}$ & $4.35 \times 10^{3}$ & $1.03 \times 10^{3}$ \\
\hline F13 & $1.34 \times 10^{3}$ & $2.12 \times 10^{3}$ & $1.66 \times 10^{3}$ & $2.27 \times 10^{2}$ & $1.34 \times 10^{3}$ & $1.88 \times 10^{3}$ & $1.57 \times 10^{3}$ & $1.66 \times 10^{2}$ \\
\hline F14 & $1.43 \times 10^{3}$ & $1.21 \times 10^{4}$ & $2.21 \times 10^{3}$ & $2.01 \times 10^{3}$ & $1.44 \times 10^{3}$ & $1.47 \times 10^{3}$ & $1.45 \times 10^{3}$ & $9.13 \times 10^{0}$ \\
\hline F15 & $1.52 \times 10^{3}$ & $1.78 \times 10^{3}$ & $1.65 \times 10^{3}$ & $8.34 \times 10^{1}$ & $1.56 \times 10^{3}$ & $2.57 \times 10^{3}$ & $1.81 \times 10^{3}$ & $2.74 \times 10^{2}$ \\
\hline F16 & $1.78 \times 10^{3}$ & $2.35 \times 10^{3}$ & $2.04 \times 10^{3}$ & $1.19 \times 10^{2}$ & $1.83 \times 10^{3}$ & $1.99 \times 10^{3}$ & $1.92 \times 10^{3}$ & $4.91 \times 10^{1}$ \\
\hline F17 & $1.77 \times 10^{3}$ & $2.07 \times 10^{3}$ & $1.92 \times 10^{3}$ & $8.16 \times 10^{1}$ & $1.78 \times 10^{3}$ & $1.92 \times 10^{3}$ & $1.87 \times 10^{3}$ & $3.70 \times 10^{1}$ \\
\hline F18 & $1.29 \times 10^{4}$ & $2.43 \times 10^{5}$ & $6.93 \times 10^{4}$ & $6.14 \times 10^{4}$ & $1.59 \times 10^{4}$ & $5.67 \times 10^{4}$ & $3.76 \times 10^{4}$ & $1.06 \times 10^{4}$ \\
\hline F19 & $1.92 \times 10^{3}$ & $3.28 \times 10^{3}$ & $2.26 \times 10^{3}$ & $3.32 \times 10^{2}$ & $1.94 \times 10^{3}$ & $4.84 \times 10^{3}$ & $2.71 \times 10^{3}$ & $8.30 \times 10^{2}$ \\
\hline F20 & $2.08 \times 10^{3}$ & $2.30 \times 10^{3}$ & $2.21 \times 10^{3}$ & $5.27 \times 10^{1}$ & $2.09 \times 10^{3}$ & $2.21 \times 10^{3}$ & $2.15 \times 10^{3}$ & $3.64 \times 10^{1}$ \\
\hline $\mathrm{F} 21$ & $2.11 \times 10^{3}$ & $2.23 \times 10^{3}$ & $2.17 \times 10^{3}$ & $2.61 \times 10^{1}$ & $2.10 \times 10^{3}$ & $2.17 \times 10^{3}$ & $2.15 \times 10^{3}$ & $3.44 \times 10^{1}$ \\
\hline $\mathrm{F} 22$ & $2.23 \times 10^{3}$ & $2.30 \times 10^{3}$ & $2.27 \times 10^{3}$ & $1.70 \times 10^{1}$ & $2.23 \times 10^{3}$ & $2.27 \times 10^{3}$ & $2.25 \times 10^{3}$ & $1.14 \times 10^{1}$ \\
\hline F23 & $2.82 \times 10^{3}$ & $2.90 \times 10^{3}$ & $2.85 \times 10^{3}$ & $1.89 \times 10^{1}$ & $2.83 \times 10^{3}$ & $2.86 \times 10^{3}$ & $2.85 \times 10^{3}$ & $1.04 \times 10^{1}$ \\
\hline F24 & $2.60 \times 10^{3}$ & $3.41 \times 10^{3}$ & $3.29 \times 10^{3}$ & $2.34 \times 10^{2}$ & $2.60 \times 10^{3}$ & $3.35 \times 10^{3}$ & $2.85 \times 10^{3}$ & $2.78 \times 10^{2}$ \\
\hline F25 & $2.90 \times 10^{3}$ & $2.99 \times 10^{3}$ & $2.93 \times 10^{3}$ & $2.92 \times 10^{1}$ & $2.90 \times 10^{3}$ & $2.92 \times 10^{3}$ & $2.92 \times 10^{3}$ & $4.89 \times 10^{0}$ \\
\hline F26 & $2.90 \times 10^{3}$ & $5.24 \times 10^{3}$ & $4.17 \times 10^{3}$ & $8.37 \times 10^{2}$ & $2.90 \times 10^{3}$ & $4.50 \times 10^{3}$ & $3.43 \times 10^{3}$ & $5.46 \times 10^{2}$ \\
\hline $\mathrm{F} 27$ & $3.45 \times 10^{3}$ & $3.56 \times 10^{3}$ & $3.49 \times 10^{3}$ & $2.65 \times 10^{1}$ & $3.46 \times 10^{3}$ & $3.54 \times 10^{3}$ & $3.51 \times 10^{3}$ & $2.26 \times 10^{1}$ \\
\hline F28 & $3.22 \times 10^{3}$ & $3.44 \times 10^{3}$ & $3.26 \times 10^{3}$ & $5.64 \times 10^{1}$ & $3.18 \times 10^{3}$ & $3.28 \times 10^{3}$ & $3.23 \times 10^{3}$ & $2.88 \times 10^{1}$ \\
\hline F29 & $3.32 \times 10^{3}$ & $3.61 \times 10^{3}$ & $3.45 \times 10^{3}$ & $7.47 \times 10^{1}$ & $3.26 \times 10^{3}$ & $3.43 \times 10^{3}$ & $3.38 \times 10^{3}$ & $5.17 \times 10^{1}$ \\
\hline F30 & $4.13 \times 10^{3}$ & $1.96 \times 10^{4}$ & $9.30 \times 10^{3}$ & $4.27 \times 10^{3}$ & $4.11 \times 10^{3}$ & $3.45 \times 10^{4}$ & $1.61 \times 10^{4}$ & $9.66 \times 10^{3}$ \\
\hline
\end{tabular}

\subsubsection{HCLPSO Based on Multi-Stage Search Strategy}

Tables 8 and 9 is the experiment data of results of HCLPSO and RaHCLPSO in the case of 10/30 dimensions according to the parameters mentioned in Section 5.2. The data in bold in the tables is preferred.

Table 8. Comparison of experimental results of HCLPSO and RaHCLPSO in the case of 10 dimensions.

\begin{tabular}{|c|c|c|c|c|c|c|c|c|}
\hline \multirow{2}{*}{ Fun } & \multicolumn{4}{|c|}{ HCLPSO (Dim = 10) } & \multicolumn{4}{|c|}{ RaHCLPSO (Dim = 10) } \\
\hline & Min & $\operatorname{Max}$ & Mean & Std & Min & $\operatorname{Max}$ & Mean & Std \\
\hline F1 & $1.07 \times 10^{2}$ & $1.53 \times 10^{3}$ & $4.30 \times 10^{2}$ & $4.29 \times 10^{2}$ & $1.00 \times 10^{2}$ & $3.48 \times 10^{2}$ & $1.75 \times 10^{2}$ & $8.54 \times 10^{1}$ \\
\hline F3 & $3.00 \times 10^{2}$ & $3.00 \times 10^{2}$ & $3.00 \times 10^{2}$ & $1.03 \times 10^{-8}$ & $3.00 \times 10^{2}$ & $3.00 \times 10^{2}$ & $3.00 \times 10^{2}$ & $5.51 \times 10^{-3}$ \\
\hline $\mathrm{F} 4$ & $4.00 \times 10^{2}$ & $4.05 \times 10^{2}$ & $4.01 \times 10^{2}$ & $1.55 \times 10^{0}$ & $4.00 \times 10^{2}$ & $4.00 \times 10^{2}$ & $4.00 \times 10^{2}$ & $1.01 \times 10^{-1}$ \\
\hline F5 & $5.02 \times 10^{2}$ & $5.12 \times 10^{2}$ & $5.06 \times 10^{2}$ & $2.29 \times 10^{0}$ & $5.02 \times 10^{2}$ & $5.05 \times 10^{2}$ & $5.05 \times 10^{2}$ & $8.56 \times 10^{-1}$ \\
\hline $\mathrm{F} 6$ & $6.00 \times 10^{2}$ & $6.00 \times 10^{2}$ & $6.00 \times 10^{2}$ & $7.44 \times 10^{-8}$ & $6.00 \times 10^{2}$ & $6.00 \times 10^{2}$ & $6.00 \times 10^{2}$ & $1.15 \times 10^{-3}$ \\
\hline F7 & $7.14 \times 10^{2}$ & $7.29 \times 10^{2}$ & $7.18 \times 10^{2}$ & $3.50 \times 10^{0}$ & $7.13 \times 10^{2}$ & $7.18 \times 10^{2}$ & $7.16 \times 10^{2}$ & $1.50 \times 10^{0}$ \\
\hline F8 & $8.02 \times 10^{2}$ & $8.09 \times 10^{2}$ & $8.06 \times 10^{2}$ & $1.97 \times 10^{0}$ & $8.02 \times 10^{2}$ & $8.06 \times 10^{2}$ & $8.05 \times 10^{2}$ & $1.18 \times 10^{0}$ \\
\hline F9 & $9.00 \times 10^{2}$ & $9.00 \times 10^{2}$ & $9.00 \times 10^{2}$ & $1.64 \times 10^{-13}$ & $9.00 \times 10^{2}$ & $9.00 \times 10^{2}$ & $9.00 \times 10^{2}$ & $4.36 \times 10^{-5}$ \\
\hline F10 & $1.01 \times 10^{3}$ & $1.38 \times 10^{3}$ & $1.14 \times 10^{3}$ & $1.16 \times 10^{2}$ & $1.00 \times 10^{3}$ & $1.14 \times 10^{3}$ & $1.05 \times 10^{3}$ & $4.50 \times 10^{1}$ \\
\hline
\end{tabular}


Table 8. Cont.

\begin{tabular}{|c|c|c|c|c|c|c|c|c|}
\hline \multirow{2}{*}{ Fun } & \multicolumn{4}{|c|}{ HCLPSO $($ Dim = 10) } & \multicolumn{4}{|c|}{ RaHCLPSO $(\mathrm{Dim}=10)$} \\
\hline & Min & Max & Mean & Std & Min & Max & Mean & Std \\
\hline F11 & $1.10 \times 10^{3}$ & $1.10 \times 10^{3}$ & $1.10 \times 10^{3}$ & $1.07 \times 10^{0}$ & $1.10 \times 10^{3}$ & $1.10 \times 10^{3}$ & $1.10 \times 10^{3}$ & $7.65 \times 10^{-1}$ \\
\hline F12 & $2.61 \times 10^{3}$ & $4.38 \times 10^{4}$ & $1.30 \times 10^{4}$ & $9.90 \times 10^{3}$ & $2.57 \times 10^{3}$ & $2.02 \times 10^{4}$ & $1.07 \times 10^{4}$ & $5.24 \times 10^{3}$ \\
\hline F13 & $1.30 \times 10^{3}$ & $1.42 \times 10^{3}$ & $1.35 \times 10^{3}$ & $3.24 \times 10^{1}$ & $1.31 \times 10^{3}$ & $1.49 \times 10^{3}$ & $1.39 \times 10^{3}$ & $5.37 \times 10^{1}$ \\
\hline F14 & $1.42 \times 10^{3}$ & $1.47 \times 10^{3}$ & $1.45 \times 10^{3}$ & $1.21 \times 10^{1}$ & $1.43 \times 10^{3}$ & $1.45 \times 10^{3}$ & $1.44 \times 10^{3}$ & $7.23 \times 10^{0}$ \\
\hline F15 & $1.51 \times 10^{3}$ & $1.56 \times 10^{3}$ & $1.52 \times 10^{3}$ & $1.13 \times 10^{1}$ & $1.51 \times 10^{3}$ & $1.53 \times 10^{3}$ & $1.52 \times 10^{3}$ & $7.12 \times 10^{0}$ \\
\hline F16 & $1.60 \times 10^{3}$ & $1.62 \times 10^{3}$ & $1.60 \times 10^{3}$ & $3.28 \times 10^{0}$ & $1.60 \times 10^{3}$ & $1.60 \times 10^{3}$ & $1.60 \times 10^{3}$ & $6.90 \times 10^{-1}$ \\
\hline F17 & $1.72 \times 10^{3}$ & $1.74 \times 10^{3}$ & $1.73 \times 10^{3}$ & $4.61 \times 10^{0}$ & $1.70 \times 10^{3}$ & $1.73 \times 10^{3}$ & $1.73 \times 10^{3}$ & $6.16 \times 10^{0}$ \\
\hline F18 & $1.93 \times 10^{3}$ & $4.12 \times 10^{3}$ & $2.62 \times 10^{3}$ & $5.66 \times 10^{2}$ & $1.89 \times 10^{3}$ & $2.69 \times 10^{3}$ & $2.31 \times 10^{3}$ & $2.63 \times 10^{2}$ \\
\hline F19 & $1.90 \times 10^{3}$ & $1.94 \times 10^{3}$ & $1.91 \times 10^{3}$ & $8.08 \times 10^{0}$ & $1.90 \times 10^{3}$ & $1.91 \times 10^{3}$ & $1.91 \times 10^{3}$ & $2.44 \times 10^{0}$ \\
\hline F20 & $2.00 \times 10^{3}$ & $2.04 \times 10^{3}$ & $2.01 \times 10^{3}$ & $1.12 \times 10^{1}$ & $2.00 \times 10^{3}$ & $2.02 \times 10^{3}$ & $2.01 \times 10^{3}$ & $9.24 \times 10^{0}$ \\
\hline F21 & $2.20 \times 10^{3}$ & $2.20 \times 10^{3}$ & $2.20 \times 10^{3}$ & $3.97 \times 10^{-6}$ & $2.10 \times 10^{3}$ & $2.26 \times 10^{3}$ & $2.23 \times 10^{3}$ & $4.20 \times 10^{1}$ \\
\hline F22 & $2.20 \times 10^{3}$ & $2.30 \times 10^{3}$ & $2.27 \times 10^{3}$ & $4.35 \times 10^{1}$ & $2.20 \times 10^{3}$ & $2.39 \times 10^{3}$ & $2.25 \times 10^{3}$ & $7.68 \times 10^{1}$ \\
\hline F23 & $2.40 \times 10^{3}$ & $2.66 \times 10^{3}$ & $2.64 \times 10^{3}$ & $4.71 \times 10^{1}$ & $2.55 \times 10^{3}$ & $2.65 \times 10^{3}$ & $2.63 \times 10^{3}$ & $2.81 \times 10^{1}$ \\
\hline F24 & $2.50 \times 10^{3}$ & $2.55 \times 10^{3}$ & $2.50 \times 10^{3}$ & $1.20 \times 10^{1}$ & $2.49 \times 10^{3}$ & $2.50 \times 10^{3}$ & $2.50 \times 10^{3}$ & $2.34 \times 10^{0}$ \\
\hline F25 & $2.89 \times 10^{3}$ & $2.90 \times 10^{3}$ & $2.90 \times 10^{3}$ & $1.14 \times 10^{0}$ & $2.89 \times 10^{3}$ & $2.90 \times 10^{3}$ & $2.90 \times 10^{3}$ & $7.35 \times 10^{-1}$ \\
\hline F26 & $2.60 \times 10^{3}$ & $2.90 \times 10^{3}$ & $2.73 \times 10^{3}$ & $1.25 \times 10^{2}$ & $2.60 \times 10^{3}$ & $2.82 \times 10^{3}$ & $2.66 \times 10^{3}$ & $9.55 \times 10^{1}$ \\
\hline F27 & $3.10 \times 10^{3}$ & $3.14 \times 10^{3}$ & $3.11 \times 10^{3}$ & $1.34 \times 10^{1}$ & $3.10 \times 10^{3}$ & $3.11 \times 10^{3}$ & $3.10 \times 10^{3}$ & $4.33 \times 10^{0}$ \\
\hline F28 & $3.08 \times 10^{3}$ & $3.15 \times 10^{3}$ & $3.10 \times 10^{3}$ & $1.33 \times 10^{1}$ & $3.08 \times 10^{3}$ & $3.10 \times 10^{3}$ & $3.10 \times 10^{3}$ & $2.75 \times 10^{0}$ \\
\hline F29 & $3.13 \times 10^{3}$ & $3.18 \times 10^{3}$ & $3.15 \times 10^{3}$ & $1.08 \times 10^{1}$ & $3.12 \times 10^{3}$ & $3.15 \times 10^{3}$ & $3.14 \times 10^{3}$ & $5.79 \times 10^{0}$ \\
\hline F30 & $3.41 \times 10^{3}$ & $1.17 \times 10^{4}$ & $5.38 \times 10^{3}$ & $2.31 \times 10^{3}$ & $3.67 \times 10^{3}$ & $5.66 \times 10^{3}$ & $4.58 \times 10^{3}$ & $5.92 \times 10^{2}$ \\
\hline
\end{tabular}

Table 9. Comparison of experimental results of HCLPSO and RaHCLPSO in the case of 30 dimensions.

\begin{tabular}{|c|c|c|c|c|c|c|c|c|}
\hline \multirow{2}{*}{ Fun } & \multicolumn{4}{|c|}{ HCLPSO $($ Dim = 30) } & \multicolumn{4}{|c|}{ RaHCLPSO (Dim = 30) } \\
\hline & Min & Max & Mean & Std & Min & Max & Mean & Std \\
\hline F1 & $1.00 \times 10^{2}$ & $1.32 \times 10^{3}$ & $4.10 \times 10^{2}$ & $3.47 \times 10^{2}$ & $1.08 \times 10^{2}$ & $8.05 \times 10^{2}$ & $3.47 \times 10^{2}$ & $2.53 \times 10^{2}$ \\
\hline F3 & $3.00 \times 10^{2}$ & $3.05 \times 10^{2}$ & $3.01 \times 10^{2}$ & $1.35 \times 10^{0}$ & $3.01 \times 10^{2}$ & $3.01 \times 10^{2}$ & $3.01 \times 10^{2}$ & $1.52 \times 10^{-1}$ \\
\hline $\mathrm{F} 4$ & $4.04 \times 10^{2}$ & $5.15 \times 10^{2}$ & $4.74 \times 10^{2}$ & $2.32 \times 10^{1}$ & $4.03 \times 10^{2}$ & $4.72 \times 10^{2}$ & $4.52 \times 10^{2}$ & $2.89 \times 10^{1}$ \\
\hline F5 & $5.19 \times 10^{2}$ & $5.58 \times 10^{2}$ & $5.37 \times 10^{2}$ & $9.26 \times 10^{0}$ & $5.17 \times 10^{2}$ & $5.36 \times 10^{2}$ & $5.29 \times 10^{2}$ & $6.47 \times 10^{0}$ \\
\hline F6 & $6.00 \times 10^{2}$ & $6.00 \times 10^{2}$ & $6.00 \times 10^{2}$ & $2.02 \times 10^{-6}$ & $6.00 \times 10^{2}$ & $6.00 \times 10^{2}$ & $6.00 \times 10^{2}$ & $8.97 \times 10^{-4}$ \\
\hline F7 & $7.52 \times 10^{2}$ & $8.16 \times 10^{2}$ & $7.83 \times 10^{2}$ & $1.70 \times 10^{1}$ & $7.61 \times 10^{2}$ & $7.90 \times 10^{2}$ & $7.79 \times 10^{2}$ & $9.24 \times 10^{0}$ \\
\hline F8 & $8.21 \times 10^{2}$ & $8.65 \times 10^{2}$ & $8.40 \times 10^{2}$ & $1.21 \times 10^{1}$ & $8.25 \times 10^{2}$ & $8.38 \times 10^{2}$ & $8.32 \times 10^{2}$ & $3.31 \times 10^{0}$ \\
\hline F9 & $9.00 \times 10^{2}$ & $9.15 \times 10^{2}$ & $9.03 \times 10^{2}$ & $4.02 \times 10^{0}$ & $9.00 \times 10^{2}$ & $9.02 \times 10^{2}$ & $9.01 \times 10^{2}$ & $5.40 \times 10^{-1}$ \\
\hline F10 & $2.06 \times 10^{3}$ & $3.92 \times 10^{3}$ & $2.91 \times 10^{3}$ & $4.45 \times 10^{2}$ & $1.96 \times 10^{3}$ & $2.65 \times 10^{3}$ & $2.43 \times 10^{3}$ & $1.87 \times 10^{2}$ \\
\hline F11 & $1.13 \times 10^{3}$ & $1.24 \times 10^{3}$ & $1.15 \times 10^{3}$ & $2.51 \times 10^{1}$ & $1.13 \times 10^{3}$ & $1.15 \times 10^{3}$ & $1.14 \times 10^{3}$ & $8.40 \times 10^{0}$ \\
\hline F12 & $2.17 \times 10^{3}$ & $1.05 \times 10^{4}$ & $4.62 \times 10^{3}$ & $1.97 \times 10^{3}$ & $8.21 \times 10^{3}$ & $2.72 \times 10^{4}$ & $1.96 \times 10^{4}$ & $5.33 \times 10^{3}$ \\
\hline F13 & $1.33 \times 10^{3}$ & $1.60 \times 10^{3}$ & $1.43 \times 10^{3}$ & $7.55 \times 10^{1}$ & $1.36 \times 10^{3}$ & $1.83 \times 10^{3}$ & $1.49 \times 10^{3}$ & $1.50 \times 10^{2}$ \\
\hline F14 & $1.45 \times 10^{3}$ & $1.60 \times 10^{3}$ & $1.52 \times 10^{3}$ & $3.89 \times 10^{1}$ & $1.47 \times 10^{3}$ & $1.59 \times 10^{3}$ & $1.53 \times 10^{3}$ & $4.29 \times 10^{1}$ \\
\hline F15 & $1.53 \times 10^{3}$ & $1.79 \times 10^{3}$ & $1.62 \times 10^{3}$ & $7.42 \times 10^{1}$ & $1.55 \times 10^{3}$ & $2.34 \times 10^{3}$ & $1.94 \times 10^{3}$ & $2.62 \times 10^{2}$ \\
\hline F16 & $1.61 \times 10^{3}$ & $2.32 \times 10^{3}$ & $1.94 \times 10^{3}$ & $1.74 \times 10^{2}$ & $1.62 \times 10^{3}$ & $1.96 \times 10^{3}$ & $1.82 \times 10^{3}$ & $1.09 \times 10^{2}$ \\
\hline F17 & $1.77 \times 10^{3}$ & $1.94 \times 10^{3}$ & $1.83 \times 10^{3}$ & $4.61 \times 10^{1}$ & $1.77 \times 10^{3}$ & $1.82 \times 10^{3}$ & $1.80 \times 10^{3}$ & $1.20 \times 10^{1}$ \\
\hline F18 & $7.96 \times 10^{3}$ & $1.13 \times 10^{5}$ & $3.49 \times 10^{4}$ & $2.69 \times 10^{4}$ & $1.14 \times 10^{4}$ & $3.66 \times 10^{4}$ & $2.68 \times 10^{4}$ & $6.88 \times 10^{3}$ \\
\hline F19 & $1.93 \times 10^{3}$ & $2.13 \times 10^{3}$ & $2.01 \times 10^{3}$ & $5.89 \times 10^{1}$ & $1.94 \times 10^{3}$ & $2.54 \times 10^{3}$ & $2.10 \times 10^{3}$ & $2.02 \times 10^{2}$ \\
\hline $\mathrm{F} 20$ & $2.06 \times 10^{3}$ & $2.27 \times 10^{3}$ & $2.15 \times 10^{3}$ & $6.79 \times 10^{1}$ & $2.06 \times 10^{3}$ & $2.14 \times 10^{3}$ & $2.10 \times 10^{3}$ & $2.43 \times 10^{1}$ \\
\hline F21 & $2.25 \times 10^{3}$ & $2.25 \times 10^{3}$ & $2.25 \times 10^{3}$ & $1.08 \times 10^{-12}$ & $2.12 \times 10^{3}$ & $2.18 \times 10^{3}$ & $2.17 \times 10^{3}$ & $1.98 \times 10^{1}$ \\
\hline F22 & $2.35 \times 10^{3}$ & $2.35 \times 10^{3}$ & $2.35 \times 10^{3}$ & $1.33 \times 10^{-12}$ & $2.22 \times 10^{3}$ & $2.24 \times 10^{3}$ & $2.23 \times 10^{3}$ & $3.85 \times 10^{0}$ \\
\hline F23 & $2.82 \times 10^{3}$ & $2.88 \times 10^{3}$ & $2.85 \times 10^{3}$ & $1.43 \times 10^{1}$ & $2.78 \times 10^{3}$ & $2.84 \times 10^{3}$ & $2.83 \times 10^{3}$ & $1.71 \times 10^{1}$ \\
\hline $\mathrm{F} 24$ & $2.60 \times 10^{3}$ & $2.60 \times 10^{3}$ & $2.60 \times 10^{3}$ & $5.28 \times 10^{-7}$ & $2.60 \times 10^{3}$ & $2.61 \times 10^{3}$ & $2.60 \times 10^{3}$ & $9.18 \times 10^{-1}$ \\
\hline $\mathrm{F} 25$ & $2.90 \times 10^{3}$ & $2.97 \times 10^{3}$ & $2.91 \times 10^{3}$ & $1.72 \times 10^{1}$ & $2.90 \times 10^{3}$ & $2.92 \times 10^{3}$ & $2.91 \times 10^{3}$ & $7.42 \times 10^{0}$ \\
\hline F26 & $2.90 \times 10^{3}$ & $2.90 \times 10^{3}$ & $2.90 \times 10^{3}$ & $3.71 \times 10^{-3}$ & $2.90 \times 10^{3}$ & $2.90 \times 10^{3}$ & $2.90 \times 10^{3}$ & $4.74 \times 10^{-3}$ \\
\hline $\mathrm{F} 27$ & $3.39 \times 10^{3}$ & $3.63 \times 10^{3}$ & $3.54 \times 10^{3}$ & $6.41 \times 10^{1}$ & $3.35 \times 10^{3}$ & $3.51 \times 10^{3}$ & $3.46 \times 10^{3}$ & $4.44 \times 10^{1}$ \\
\hline F28 & $3.14 \times 10^{3}$ & $3.30 \times 10^{3}$ & $3.23 \times 10^{3}$ & $3.42 \times 10^{1}$ & $3.14 \times 10^{3}$ & $3.22 \times 10^{3}$ & $3.19 \times 10^{3}$ & $2.94 \times 10^{1}$ \\
\hline F29 & $3.21 \times 10^{3}$ & $3.41 \times 10^{3}$ & $3.29 \times 10^{3}$ & $5.57 \times 10^{1}$ & $3.26 \times 10^{3}$ & $3.30 \times 10^{3}$ & $3.28 \times 10^{3}$ & $1.33 \times 10^{1}$ \\
\hline F30 & $4.22 \times 10^{3}$ & $1.56 \times 10^{4}$ & $7.68 \times 10^{3}$ & $3.06 \times 10^{3}$ & $9.50 \times 10^{3}$ & $3.57 \times 10^{4}$ & $2.69 \times 10^{4}$ & $7.65 \times 10^{3}$ \\
\hline
\end{tabular}

\section{Analysis of the Experimental Results}

Figure 9 shows various test functions of more significant optimization effects from the six tables in Section 5.3. The $x$-axis is the fitness evaluation number (Fes, 10 dimensions equal $10^{5} ; 30$ dimensions equal $3 \times 10^{5}$ ). The $y$-axis is the average fitness value. Different colors represent different original algorithms. Solid lines represent algorithms with the strategy and dotted lines represent those without. According to the figure, algorithms adopting the strategy achieve lower (better) fitness and better search performance. Since the strategy increases population disorder during early iterations, the phenomena of slower convergence in early iterations and a poorer fitness value could be observed in certain 
circumstances in the figure; however, these phenomena are often improved at the end of search. Full development in the preliminary stage lays a more solid foundation for later exploration, and may in turn yield better fitness values. According to Figure 9, the red dotted curve of PSO is always the first stabilized, which means that PSO is more likely to be trapped in local optima. Both TSLPSO and HCLPSO can improve this. However, the assistance of the new strategy can produce better results. According to the figure, the strategy has a more significant optimizing effect on PSO and its two improved algorithms. The same conclusion is drawn in 10 dimensions and 30 dimensions, which further verifies the effectiveness of the strategy as regards the scope of the application.

Function $1(\operatorname{Dim}=10)$

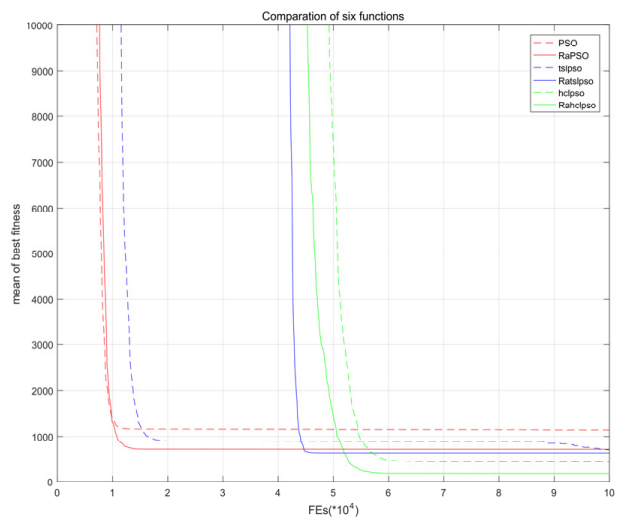

Function $7(\operatorname{Dim}=10)$

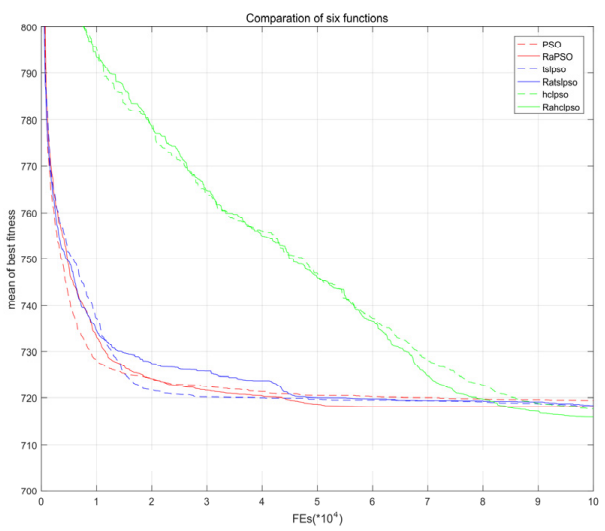

Function $14(\operatorname{Dim}=10)$

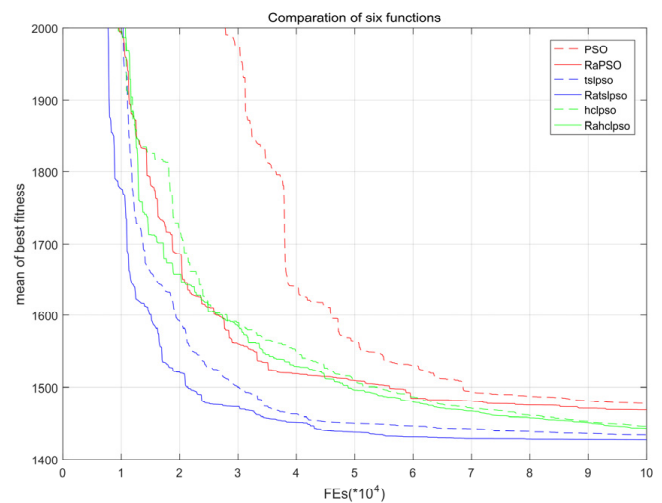

Function $5($ Dim $=10)$

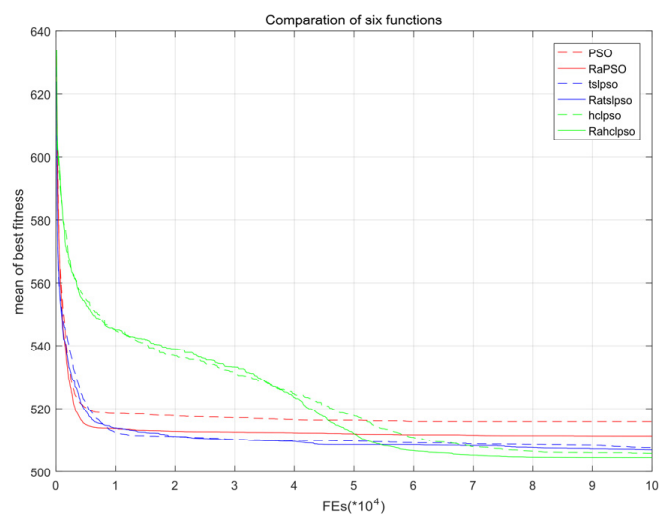

Function $11(\operatorname{Dim}=10)$

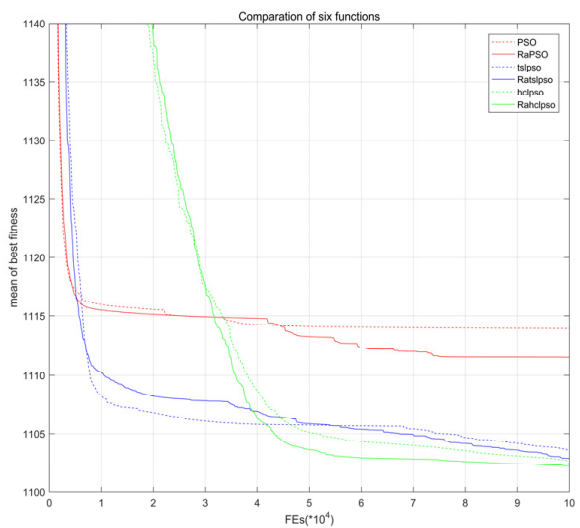

Function $15(\operatorname{Dim}=10)$

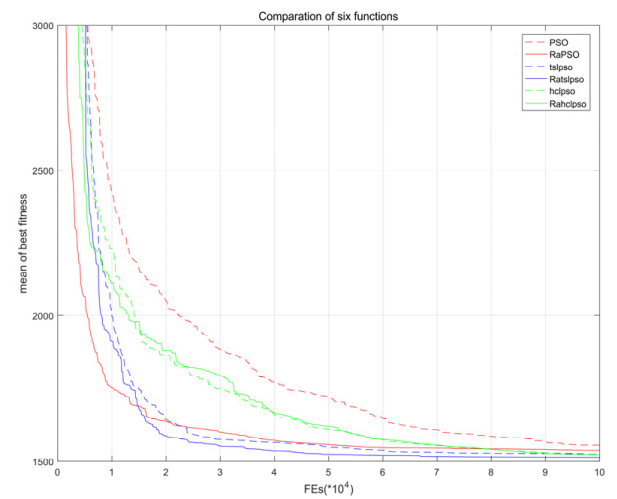

Figure 9. Cont. 
Function $24(\operatorname{Dim}=10)$

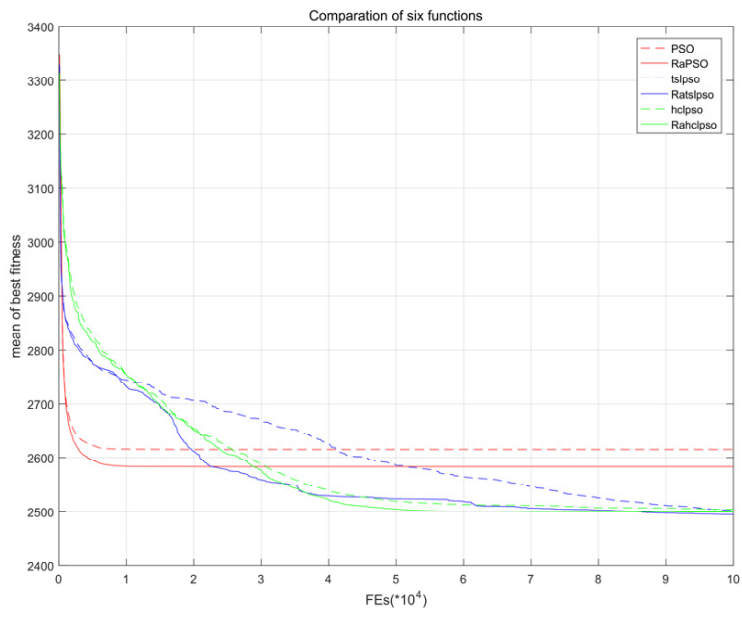

Function $4(\operatorname{Dim}=30)$

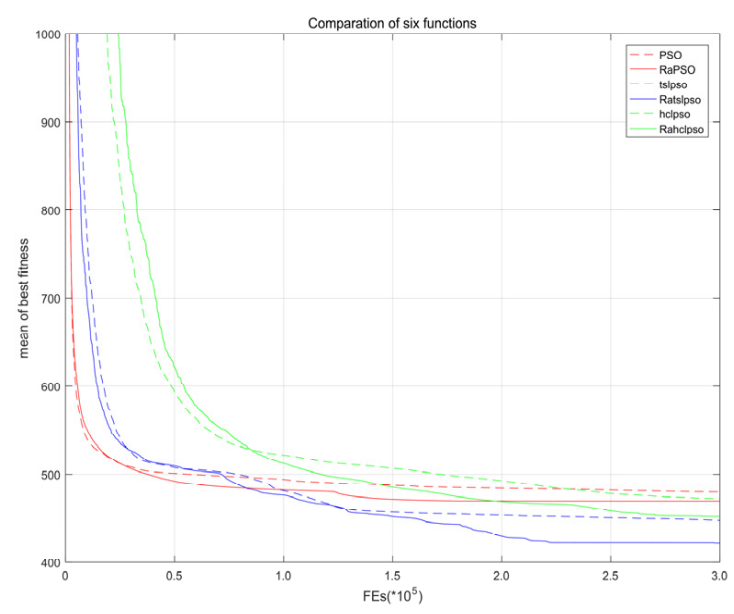

Function $9(\operatorname{Dim}=30)$

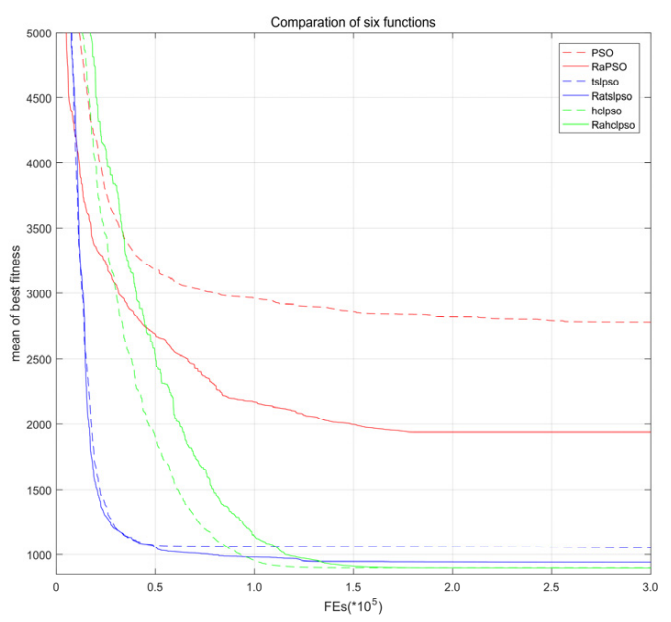

Function $26(\operatorname{Dim}=10)$

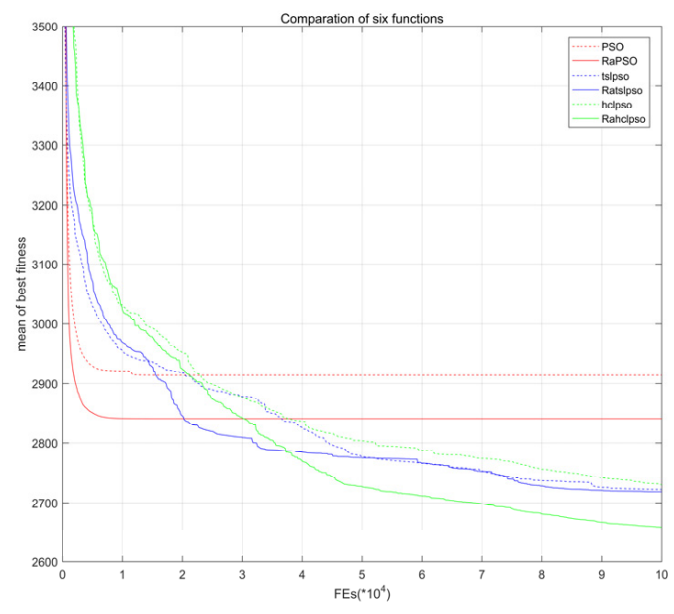

Function $6(\operatorname{Dim}=30)$

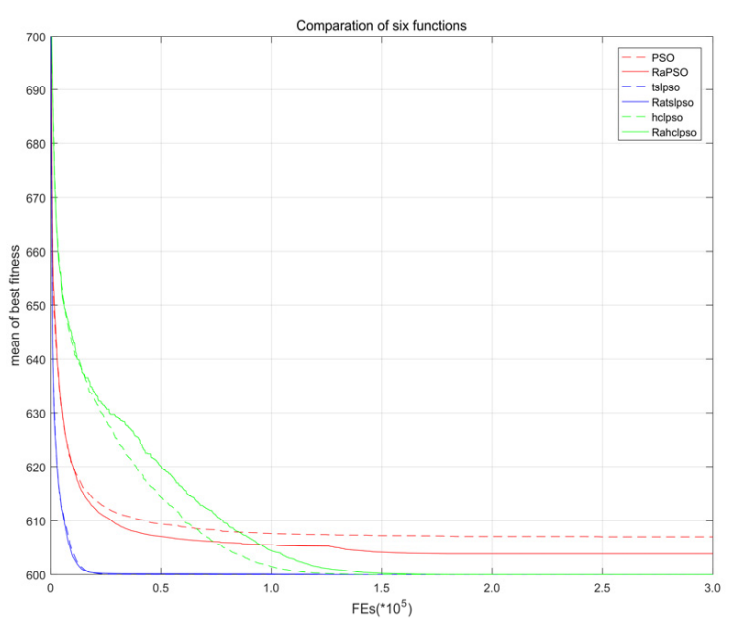

Function $10(\mathrm{Dim}=30)$

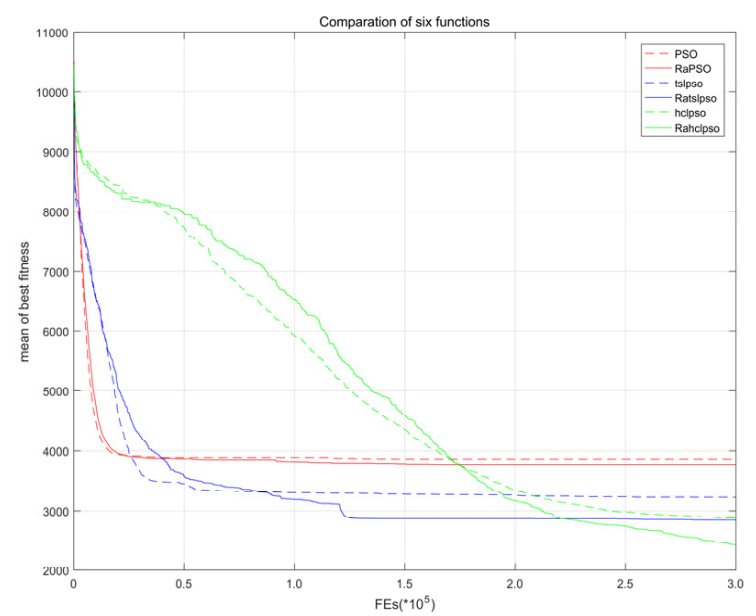

Figure 9. Cont. 
Function $16(\operatorname{Dim}=30)$

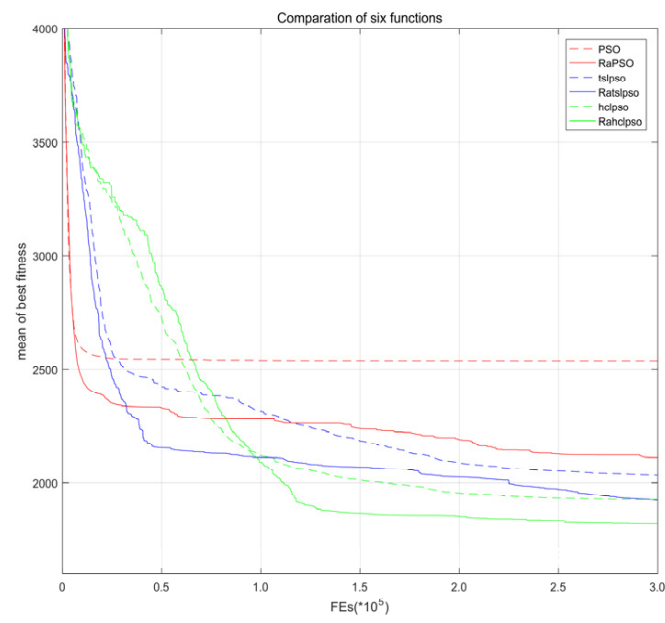

Function $25(\operatorname{Dim}=30)$

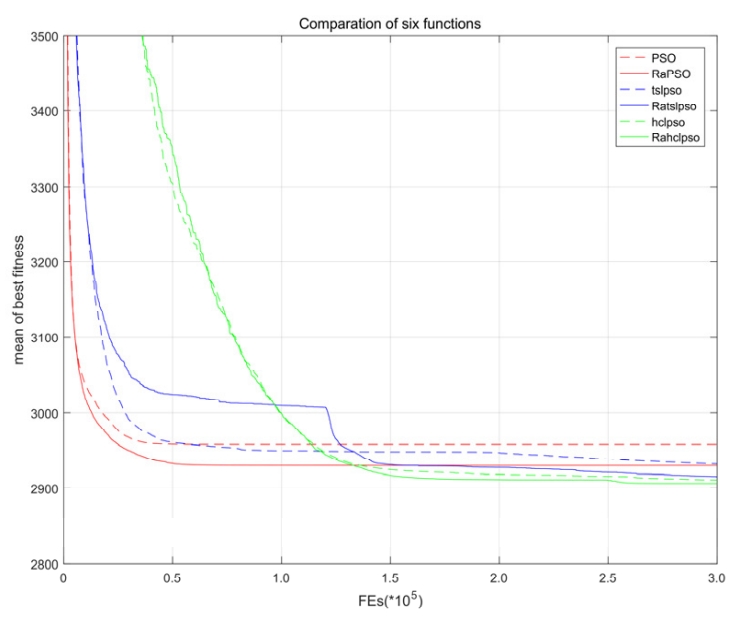

Function $20(\operatorname{Dim}=30)$

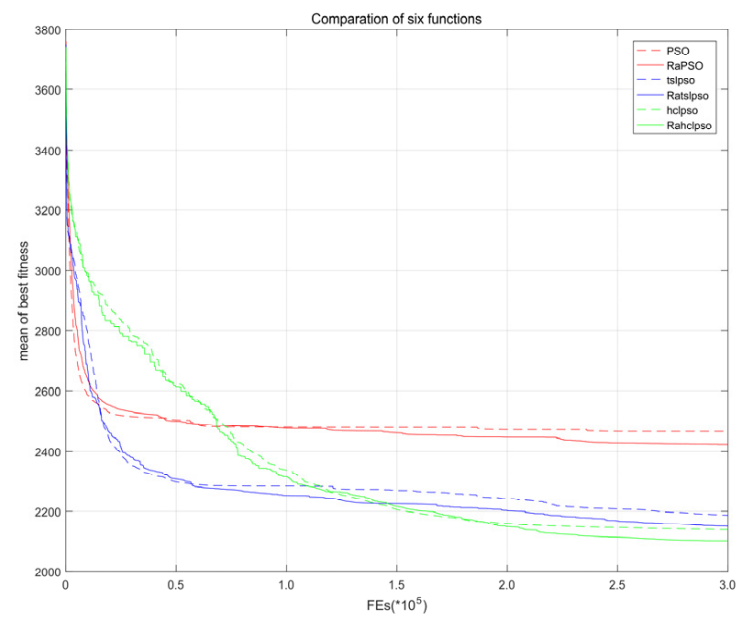

Function $29(\operatorname{Dim}=30)$

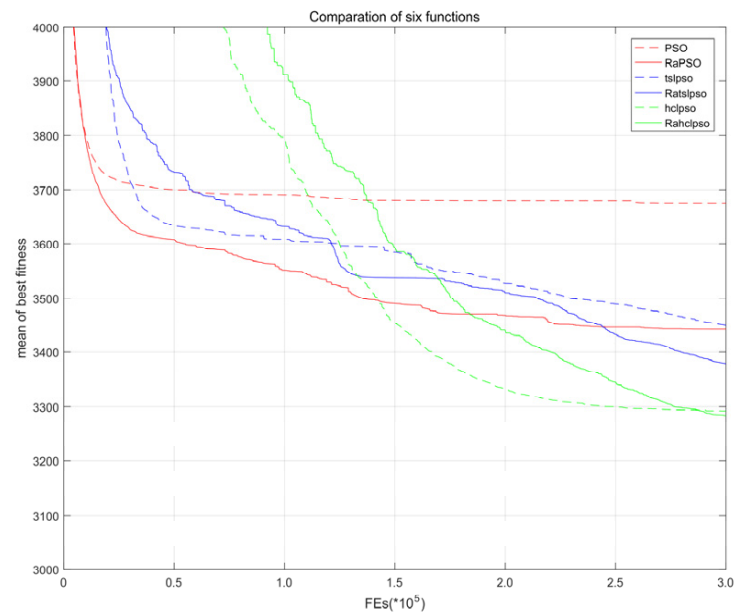

Figure 9. Comparison of the six algorithms.

\section{Conclusions}

A relatively direct and simple method is proposed herein to address problems such as premature convergence and poor searching precision with PSO, i.e., improving particle swarm diversity in early iterations of the algorithm and introducing guidance for the best particle location in the population at the end of the iteration to enhance searching precision. The concept was based on PSO and its two improved algorithms and acts as a multi-stage search strategy operator. It guides the particle swarm to different goals in different stages, improves the comprehensive exploration and development ability, and upgrades population disorder without changing the convergence performance of the algorithm too much, thus effectively improving the particle's ability to jump out of local optima. Particle swarm behavior before and after the application of the strategy was compared and described, and the effectiveness of the strategy was verified with the population entropy and DBscan tools. The contrast experiment indicated the selection process for three parameters in the proposed strategy. Moreover, according to experimental results of the algorithms on the test functions, the strategy can improve search performance.

In future work, we hope to mathematically demonstrate that the selected parameter values are optimal, prove the general applicability of the strategy, and demonstrate the 
adequacy and necessity of the divided stages. The effectiveness of the proposed strategy was experimentally demonstrated, but a mathematical analysis and proof are required.

Author Contributions: Conceptualization, Y.S.; methodology, Y.S.; project administration, W.C. and H.K.; software, X.S.; validation, X.S. and Q.C.; visualization, W.C. and H.Z.; formal analysis, Y.S.; investigation, H.Z.; resources H.K.; data curation, W.C.; writing-original draft preparation, W.C.; writing-review and editing, Y.S. and H.Z.; supervision, X.S.; funding acquisition, Y.S. All authors have read and agreed to the published version of the manuscript.

Funding: This research was funded by the National Natural Science Foundation of China, grant number 61663046, 61876166, and by the Open Foundation of Key Laboratory of Software Engineering of Yunnan Province, grant number 2020SE308, 2020 SE309.

Institutional Review Board Statement: Not applicable.

Informed Consent Statement: Not applicable.

Data Availability Statement: Not applicable.

Conflicts of Interest: The authors declare no conflict of interest.

\section{References}

1. Hosseinabadi, A.A.R.; Vahidi, J.; Saemi, B.; Sangaiah, A.K.; Elhoseny, M. Extended Genetic Algorithm for solving open-shop scheduling problem. Soft Comput. 2018, 23, 5099-5116. [CrossRef]

2. Xue, Y.; Jiang, J.; Zhao, B.; Ma, T. A self-adaptive artificial bee colony algorithm based on global best for global optimi-zation. Soft Comput. 2018, 22, 2935-2952. [CrossRef]

3. Li, W.; Li, S.; Chen, Z.; Zhong, L.; Ouyang, C. Self-feedback differential evolution adapting to fitness landscape characteristics. Soft Comput. 2017, 23, 1151-1163. [CrossRef]

4. Wei, L.; Zhang, Z.; Zhang, D.; Leung, S.C. A simulated annealing algorithm for the capacitated vehicle routing problem with two-dimensional loading constraints. Eur. J. Oper. Res. 2018, 265, 843-859. [CrossRef]

5. Engin, O.; Güçlü, A. A new hybrid ant colony optimization algorithm for solving the no-wait flow shop scheduling problems. Appl. Soft Comput. 2018, 72, 166-176. [CrossRef]

6. Yadav, S.; Ekbal, A.; Saha, S. Feature selection for entity extraction from multiple biomedical corpora: A PSO-based approach. Soft Comput. 2018, 22, 6881-6904. [CrossRef]

7. Eberhart, R.; Kennedy, J. A new optimizer using particle swarm theory[C]//MHS'95. In Proceedings of the Sixth International Symposium on Micro Machine and Human Science, Nagoya, Japan, 4-6 October 1995; pp. 39-43.

8. Han, Y.S.; Zhang, L.; Tan, H.Y.; Xue, X.L. Mobile robot path planning based on improved particle swarm optimization algorithm. J. Xi'an Polytech. Univ. 2019, 33, 517-523. (In Chinese)

9. Yang, Y. Research on image segmentation algorithm with dynamic particle swarm optimization k-means. Modern Comput. 2019, 8, 63-67. (In Chinese)

10. Zhao, H.W.; Li, S.P. Research on resources scheduling method in cloud computing based on PSO and RBF neural network. Comput. Sci. 2016, 43, 113-117. (In Chinese)

11. Liu, H.; Shen, X.; Qu, H.; Wang, P. Neural network pid biogas dry fermentation temperature control by particle swarm optimization. Comput. Eng. Design 2017, 38, 784-788.

12. Sun, Q.; Ren, S.; Le, Y.G. Research on data prediction model based on particle swarm optimization extreme learning machine. J. Sichuan Inst. Technol. (Nat. Sci. Ed. ) 2019, 32, 35-41. (In Chinese)

13. Zhou, X.; Cheng, X.; Peng, W.; Sun, L.; Lu, J. A particle swarm optimization algorithm-based noise control method for cooling tower clusters. J. Xi'an Univ. Eng. 2019, 33, 568-574.

14. Gunasundari, S.; Janakiraman, S.; Meenambal, S. Velocity bounded boolean particle swarm optimization for improved feature selection in liver and kidney disease diagnosis. Expert Syst. 2016, 56, 28-47. [CrossRef]

15. Marandi, A.; Afshinmanesh, F.; Shahabadi, M.; Bahrami, F. Boolean Particle Swarm Optimization and Its Application to the Design of a Dual-Band Dual-Polarized Planar Antenna. In Proceedings of the 2006 IEEE International Conference on Evolutionary Computation, Vancouver, BC, Canada, 16-21 July 2006. [CrossRef]

16. Sen, T.; Pragallapati, N.; Agarwal, V.; Kumar, R. Global maximum power point tracking of PV arrays under partial shading conditions using a modified particle velocity-based PSOtechnique, IET Renew. Power Gener. 2017, 12, 555-564. [CrossRef]

17. Shi, Y.; Eberhart, R. A modified particle swarm optimizer. In Proceedings of the 1998 IEEE International Conference on Evolutionary Computation Proceedings (IEEE World Congress on Computational Intelligence), (Cat. No.98TH8360). Anchorage, AK, USA, 4-9 May 1998; 73, pp. 69-73. [CrossRef]

18. Ratnaweera, A.; Halgamuge, S.; Watson, H.C. Self-Organizing Hierarchical Particle Swarm Optimizer with Time-Varying Acceleration Coefficients. IEEE Trans. Evol. Comput. 2004, 8, 240-255. [CrossRef] 
19. Zhan, Z.H.; Zhang, J.; Li, Y.; Chung, H.S.H. Adaptive particle swarm optimization. IEEE Trans. Syst. Man Cybern. Part B Cybern. 2009, 39, 1362-1381. [CrossRef] [PubMed]

20. Chen, K.; Zhou, F.Y.; Liu, A.L. Chaotic dynamic weight particle swarm optimization for numerical function optimiza-tion. Knowl. Based Syst. 2018, 139, 23-40. [CrossRef]

21. Liang, J.; Suganthan, P. Dynamic multi-swarm particle swarm optimizer. In Proceedings of the IEEE Swarm Intelligence Symposium, Pasadena, CA, USA, 8-10 June 2005; pp. 124-129.

22. Liu, H.R.; Cui, J.C.; Lu, Z.D.; Liu, D.Y.; Deng, Y.J. A hierarchical simple particle swarm optimization with mean dimen-sional information. Appl. Soft Comput. 2019, 76, 712-725. [CrossRef]

23. Zhan, Z.-H.; Zhang, J.; Li, Y.; Shi, Y.-H. Orthogonal Learning Particle Swarm Optimization. IEEE Trans. Evol. Comput. 2010, 15, 832-847. [CrossRef]

24. Xu, G.; Cui, Q.; Shi, X. Particle Swarm Optimization based on dimensional learning Strategy. Swarm Evol. Comput. 2019, 45, 33-51. [CrossRef]

25. Li, W.; Meng, X.; Huang, Y.; Fu, Z.-H. Multipopulation cooperative particle swarm optimization with a mixed mutation strategy. Inf. Sci. 2020, 529, 179-196. [CrossRef]

26. Kennedy, J.; Mendes, R. Population structure and particle swarm performance. In Proceedings of the 2002 Congress on Evolutionary Computation, CEC02 (Cat. No.02TH8600). Honolulu, HI, USA, 12-17 May 2002; pp. 1671-1676.

27. Mendes, R.; Kennedy, J.; Neves, J. The Fully Informed Particle Swarm: Simpler, Maybe Better. IEEE Trans. Evol. Comput. 2004, 8 , 204-210. [CrossRef]

28. Janson, S.; Middendorf, M. A hierarchical particle swarm optimizer and its adaptive variant. IEEE Trans. Syst. Man, Cybern. Part $B$ Cyber. 2005, 35, 1272-1282. [CrossRef]

29. Zhang, X.; Wang, X.; Kang, Q.; Cheng, J. Differential mutation and novel social learning particle swarm optimization algorithm. Inf. Sci. 2019, 480, 109-129. [CrossRef]

30. Nasiraghdam, M.; Nafar, M. New Approach Based on Hybrid GA and PSO as HGAPSO in Low-Frequency Oscillation Damping Using UPFC Controller. J. Basic Appl. Sci. Res. 2011, 1, 2208-2218.

31. Wang, F.; Zhu, H.; Li, W.; Li, K. A hybrid convolution network for serial number recognition on banknotes. Inf. Sci. 2019, 512, 952-963. [CrossRef]

32. Meng, A.; Li, Z.; Yin, H.; Chen, S.; Guo, Z. Accelerating particle swarm optimization using crisscross search. Inf. Sci. 2016, 329, 52-72. [CrossRef]

33. Gong, Y.-J.; Li, J.-J.; Zhou, Y.; Li, Y.; Chung, H.; Shi, Y.-H.; Zhang, J. Genetic Learning Particle Swarm Optimization. IEEE Trans. Cybern. 2015, 46, 2277-2290. [CrossRef]

34. Bergh, F.V.D.; Engelbrecht, A. A Cooperative Approach to Particle Swarm Optimization. IEEE Trans. Evol. Comput. 2004, 8, 225-239. [CrossRef] 